City University of New York (CUNY) CUNY Academic Works

2016

\title{
Locally anisotropic toposes
}

Jonathon Funk

CUNY Queensborough Community College

Pieter Hofstra

University of Ottawa

\section{How does access to this work benefit you? Let us know!}

More information about this work at: https://academicworks.cuny.edu/qb_pubs/59

Discover additional works at: https://academicworks.cuny.edu

This work is made publicly available by the City University of New York (CUNY).

Contact: AcademicWorks@cuny.edu 


\title{
Locally anisotropic toposes
}

\author{
Jonathon Funk \\ Department of Mathematics and Computer Science \\ Queensborough Community College, CUNY \\ 222-05 56th Avenue Bayside, NY 11364, United States \\ Pieter Hofstra* \\ Department of Mathematics and Statistics \\ University of Ottawa \\ 585 King Edward Avenue, Ottawa, ON, K1N 6N5, Canada
}

\begin{abstract}
This paper continues the investigation of isotropy theory for toposes. We develop the theory of isotropy quotients of toposes, culminating in a structure theorem for a class of toposes we call locally anisotropic. The theory has a natural interpretation for inverse semigroups, which clarifies some aspects of how inverse semigroups and toposes are related.

Keywords: Topos theory, Inverse semigroups

2010 MSC: 18B25, 18B40, 20M18
\end{abstract}

\section{Introduction}

Every Grothendieck topos has internal to it a canonical group object $Z$ called its isotropy group [5]. This group classifies isotropy in the sense that for any object $X$ of a topos $\mathscr{E}$, morphisms $X \rightarrow Z$ of $\mathscr{E}$ are in natural bijection with automorphisms of the so-called étale geometric morphism

$$
\mathscr{E} / X \longrightarrow \mathscr{E}
$$

associated with $X$. An automorphism of this geometric morphism is given by a natural automorphism of its inverse image functor

$$
-\times X: \mathscr{E} \longrightarrow \mathscr{E} / X
$$

\footnotetext{
*Corresponding Author

Email address: phofstra@uottawa.ca (Pieter Hofstra)
} 
or equivalently by a natural automorphism of its left adjoint

$$
\Sigma_{X}: \mathscr{E} / X \longrightarrow \mathscr{E}
$$

If $Z$ is the trivial group, then we say that the topos $\mathscr{E}$ is anisotropic. If $\mathscr{E} / X$ is anisotropic, then we say that $X$ is anisotropic.

An important property of the isotropy group $Z$ is that every object $X$ of $\mathscr{E}$ carries a canonical action by it, which we denote

$$
\theta_{X}: X \times Z \rightarrow X
$$

Moreover, every morphism of $\mathscr{E}$ is equivariant with respect to this action, and $Z$ acts on itself by conjugation. It happens that this universal action by $Z$ is terminal with these properties [5].

Let us say that an object $X$ is isotropically trivial if its universal action $\theta_{X}$ by $Z$ is trivial. The full subcategory $\mathscr{E}_{\theta}$ of all such isotropically trivial objects is a topos: its inclusion in $\mathscr{E}$ is the inverse image functor of a connected, atomic geometric morphism

$$
\psi: \mathscr{E} \longrightarrow \mathscr{E}_{\theta}
$$

we call the isotropy quotient of $\mathscr{E}$.

\subsection{Objectives}

Our first objective is to analyse the isotropy quotient (1) beginning with the question: when is it equivalent to $\mathcal{B}(\mathscr{F} ; G) \longrightarrow \mathscr{F}$ for a group $G$ internal to an anisotropic topos $\mathscr{F}$, where $\mathcal{B}(\mathscr{F} ; G)$ denotes the topos of right $G$-objects in $\mathscr{F}$ ? We find that in this case the isotropy is split in a sense that we will clarify later. The analysis unfolds as a semi-direct product decomposition, not unlike what occurs in homological algebra.

We broaden the question by generalizing split isotropy to locally split isotropy. Consequently, we seek to study toposes that are locally anisotropic in the sense that they have a globally supported anisotropic object. (This bears some analogy to localic and locally localic, i.e., étendue.) Locally split isotropy quotients are related to what we shall call isotropy algebras: by this we mean an atomic geometric morphism $\mathscr{F} \stackrel{\varphi}{\longrightarrow} \mathscr{E}$ together with a section of the canonical homomorphism $m: Z_{\mathscr{F}} \rightarrow \varphi^{*} Z_{\mathscr{E}}$ that compares the isotropy groups of $\mathscr{E}$ and $\mathscr{F}$ along $\varphi$.

Our final aim is to interpret the theory for toposes typically associated with inverse semigroups. Such a topos is an étendue, whence locally anisotropic; however, we observe that the concept of locally anisotropic toposes offers a broader perspective that is relevant to the particular aspects of inverse semigroups that we shall explain. 


\subsection{Main contributions}

1. We introduce and study isotropy torsors, and show that such structures are classified by étale splittings of the isotropy quotient (Proposition 4.11, Theorem 4.12).

2. Generalizing from torsors to well-supported anisotropic objects we prove a structure theorem for locally anisotropic toposes, which states that a topos is locally anisotropic if and only if it can be covered

$$
\mathscr{E} / O \longrightarrow \mathscr{E}
$$

by an isotropically trivial object $O$, such that $\mathscr{E} / O \simeq \mathcal{B}(\mathscr{F} ; G)$ for some group $G$ internal to an anisotropic topos $\mathscr{F}$ (Theorem 4.16).

3. In the process of proving the structure theorem we develop a significant amount of general theory that includes some results worth mentioning for their own sake. One such result that we frequently put to work in our argumentation is a calculation of the isotropy group of $\mathcal{B}(\mathscr{E} ; G)$, where $G$ is a group internal to a topos $\mathscr{E}$, as the semidirect product of the isotropy group of $\mathscr{E}$ with $G$ (Theorem 3.9). Another is the 'fundamental lemma' of isotropy theory (Lemma 4.3), which is a basic fact about the behaviour of isotropy quotients with respect to slicing.

4. We show how free isotropy algebras are closely related to the structure theorem. Indeed, every object $X$ of a topos has associated with it a free isotropy algebra whose underlying geometric morphism we denote $\widehat{X}[5]$. We observe that $X$ is anisotropic if and only if $\widehat{X}$ is isotropically trivial, in the sense that its inverse image functor preserves the isotropy group. Moreover, we show that in this case $\widehat{X}$ is étale: it is equivalent to the isotropically trivial map $\mathscr{E} / O_{X} \rightarrow \mathscr{E}$, where $O_{X}$ denotes the orbit space of $X$ by the universal action of the isotropy group $Z$. When $X$ has global support, the map above is shown to be the isotropically trivial cover (2) given by the structure theorem.

5. We provide a detailed explanation about how the theory is interpreted for inverse semigroups. In topos theory, the existence of an étale section of the isotropy quotient of a topos $\mathscr{E}$ is equivalent to $\mathscr{E} \simeq \mathcal{B}(\mathscr{F} ; G)$ for $G$ internal to anisotropic $\mathscr{F}$. Billhardt theory for inverse semigroups, which says that the existence of a splitting of the maximum idempotent-separating congruence of an inverse semigroup $S$ is equivalent to $S \cong T \ltimes G$ for $G$ a $T$-group with $T$ fundamental [9], is in fact a special case of the topos theory results. Consequently, isotropy torsors constitute another equivalent approach to the Billhardt theory of congruences on semigroups. 


\subsection{Organization of the paper}

We begin with a discussion of geometric morphisms of the form $\mathcal{B}(\mathscr{E} ; G) \longrightarrow \mathscr{E}$ and their properties $(\S 2.1)$. In $\S 2.2$ we explain why such geometric morphisms are characterized (amongst all connected atomic ones) by their property that they have an étale section. For our purposes, we need the more inclusive notion of a locally split geometric morphism, which we review in $\S 2.3$.

In $\S 3$ we first review the basic definitions of isotropy theory, then in $\S 3.2$ give an explicit description of the isotropy group of a slice topos, and specialize this in $\S 3.3$ to the case where we slice by the isotropy group itself. We calculate in $\S 3.4$ the isotropy group of toposes of the form $\mathcal{B}(\mathscr{E} ; G)$ for a group $G$ internal to a topos $\mathscr{E}$.

The central results of the paper can be found in $\S 4$. The fundamental lemma of isotropy and some of its consequences ( $\S 4.1$ ) are basic tools we need to establish the structure theorem for toposes with split isotropy ( $\S 4.2)$. Its generalization to locally anisotropic toposes follows in $\S 4.4$; this section also discusses the interpretation of the result in terms of isotropy algebras.

In $\S 5$ we apply the results to the theory of inverse semigroups. We show that isotropy torsors over an inverse semigroup and (central isomorphism classes of) homomorphic sections of the so-called fundamental quotient of the inverse semigroup ( $\S 5.2$ ). The structure theorem for locally anisotropic toposes predicts that the topos of the centralizer of the idempotents $Z(E) \subseteq S$ of an inverse semigroup $S$ is equivalent to a slice topos of the topos of $S(\S 5.3)$.

We have in mind a reader with at least a basic knowledge of the theory of Grothendieck toposes and geometric morphisms, even though we do include the definitions of some of the important classes of geometric morphisms needed for the paper ( $\S 2.1$ ). All toposes in this paper are Grothendieck toposes. From time to time we also make use of the internal language of a topos mainly in order to facilitate proofs and simplify notation. The reader may wish to consult the literature for a thorough explanation of this language $[6,10]$.

\section{Atomic geometric morphisms}

We explain some of the theory of (locally split) atomic geometric morphisms, ultimately characterizing morphisms of the form (3). This material is mostly known [6], but we include it because the details are important, in order to keep the paper self-contained, and to fix notation and terminology.

\subsection{Group actions}

Johnstone [6] provides a thorough explanation of atomic geometric morphisms and their various characterizations, but let us review some of the basics here. A geometric morphism $\psi: \mathscr{F} \longrightarrow \mathscr{E}$ is called atomic when its inverse image functor $\psi^{*}: \mathscr{E} \longrightarrow \mathscr{F}$ preserves power objects $\Omega^{X}$, where $\Omega$ denotes the subobject classifier of $\mathscr{E}$. In fact, 
such a $\psi^{*}$ is logical in the sense that it must preserves all of the topos structure (finite limits/colimits, exponentials and the subobject classifier).

The class of atomic geometric morphisms is closed under composition and pullback, and includes all étale morphisms (local homeomorphisms) $\mathscr{E} / X \longrightarrow \mathscr{E}$. An atomic geometric morphism is locally connected in the sense that $\psi^{*}$ has a further $\mathscr{E}$-indexed left adjoint $\psi_{!}$. In particular, this implies that $\psi^{*}$ preserves exponentials. Intuitively, $\psi_{!}(Y)$ is the object of connected components of $Y$. If $\psi$ is atomic, then the connected objects are in fact atoms in the sense that they possess no non-trivial subobjects.

A connected geometric morphism is one whose inverse image functor is full and faithful. It follows that a locally connected morphism $\psi$ is connected if and only if $\psi_{!}(1)=1$. A locally connected morphism $\psi: \mathscr{F} \longrightarrow \mathscr{E}$ may be factored (essentially uniquely) as a composite

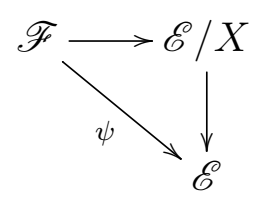

where the first factor is connected, locally connected. The object $X$ is uniquely determined as $\psi_{!}(1)$. If $\psi$ is atomic, then the first factor is atomic as well.

The (connected) atomic geometric morphism

$$
\gamma: \mathcal{B}(\mathscr{E} ; G) \longrightarrow \mathscr{E}
$$

that arises from a group action is important for our purposes. Throughout, if $G$ is a group object of a topos $\mathscr{E}$, then $\mathcal{B}(\mathscr{E} ; G)$ denotes the topos of (right) $G$-objects; its objects are objects $X$ of $\mathscr{E}$ equipped with an action $\mu: X \times G \rightarrow X$ of $G$ on $X$, and its morphisms are morphisms of $\mathscr{E}$ that respect the action. Sometimes we omit reference to the action map $\mu$, writing $\mu(x, g)=x g$, and $(X, \mu)$ simply as $X$. An important object of $\mathcal{B}(\mathscr{E} ; G)$ is 'Yoneda' $G$, which is the group itself with right multiplication. The inverse image functor of (3) equips an object of $\mathscr{E}$ with its trivial action. The left adjoint $\gamma_{\text {! }}$ is given by the coequalizer

$$
X \times G \stackrel{\mu}{\longrightarrow} X \longrightarrow X / \mu
$$

of the action and the projection: we also refer to $X / \mu$ as the orbit space of $(X, \mu)$. The atomic geometric morphism (3) is also connected, and it has a section

$$
p: \mathscr{E} \longrightarrow \mathcal{B}(\mathscr{E} ; G)
$$

given by the unit $1 \rightarrow G$ of the group. Explicitly, $p$ consists of

$$
p^{*}(X, \mu)=X ; \quad p_{!}(Y)=\gamma^{*}(Y) \times G ; \quad p_{*}(Y)=\gamma^{*}(Y)^{G} .
$$


The group $G$ is recovered as $G \cong \gamma_{*}\left(G^{G}\right) \cong \gamma_{!}(G \times G)$. Also note that the section $p$ is étale, and a surjection in the sense that $p^{*}$ is a faithful functor. In fact, we have a commutative triangle

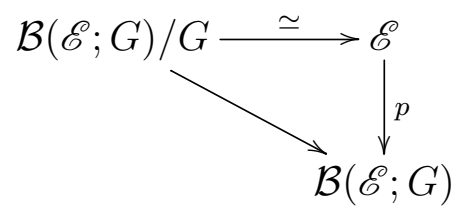

where the horizontal map is an equivalence. This is a special case of Lemma 2.2 below, but in this case one direction of the equivalence sends an object $X \stackrel{f}{\rightarrow} G$ to $X / \mu$. The inverse functor sends $Y$ to $\gamma^{*} Y \times G \rightarrow G$. To say that these functors form an equivalence is therefore to say that every such $f$ decomposes as a coproduct of copies of $G$ : the canonical comparison map

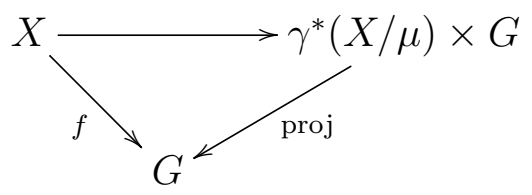

which, in set-theoretic notation, sends $x \in X$ to the pair $([x], f(x))$, where $[x]$ denotes the orbit of $x$ under the $G$-action, is an isomorphism.

In $\oint 2.2$ we will also employ the fact that $\mathcal{B}(\mathscr{E} ; G)$ is monadic over $\mathscr{E}$ for a monad whose underlying functor is $-\times G$, and whose unit and multiplication come from the unit and multiplication of the group $G$.

\section{2. Étale sections}

Johnstone explains that connected atomic maps that have a section correspond to localic groups ([6], Theorem C5.2.13). Our focus is when the section is étale as this is also sufficient for atomic morphisms $(3)$ in the sense that any connected atomic $\mathscr{F} \longrightarrow \mathscr{E}$ that has an étale section must have the form (3).

Suppose that a geometric morphism $\psi: \mathscr{F} \longrightarrow \mathscr{E}$ has a locally connected section $p$. For the moment, no assumptions on $\psi$ are made. Let $X$ denote the object $p_{!}(1)$ of $\mathscr{F}$. Any naturality square

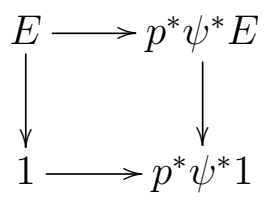

is a pullback because the horizontal maps are isomorphisms, so that by the strength of $p_{!} \dashv p^{*}$ its transpose

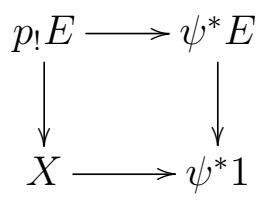


is a pullback as well. This means that there is a natural isomorphism

$$
p_{!} E \cong \psi^{*} E \times X .
$$

Therefore, for any $E$ of $\mathscr{E}$ and $F$ of $\mathscr{F}$, we have a sequence of natural bijections

$$
\begin{aligned}
& \frac{E \rightarrow p^{*} F}{p_{!} E \rightarrow F} \\
& \frac{\overline{\psi^{*} E \times X \rightarrow F}}{\frac{\psi^{*} E \rightarrow F^{X}}{E \rightarrow \psi_{*}\left(F^{X}\right)}}
\end{aligned}
$$

and hence that

$$
p^{*}(F) \cong \psi_{*}\left(F^{X}\right)
$$

for every object $F$ of $\mathscr{F}$.

Lemma 2.1. A locally connected section is necessarily étale.

Proof. Suppose that $p: \mathscr{E} \longrightarrow \mathscr{F}$ is a locally connected section of a geometric morphism $\psi$. It suffices to prove that for every object $E$ of $\mathscr{E}$, the adjunction square

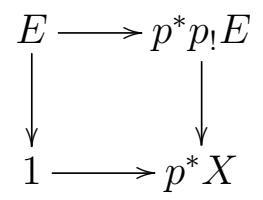

is a pullback, where $X=p_{!}(1)$. However, by (4) we have

$$
p^{*} p_{!} E \cong p^{*}\left(\psi^{*} E \times X\right) \cong p^{*} \psi^{*} E \times p^{*} X \cong E \times p^{*} X .
$$

This means that the adjunction square is isomorphic to

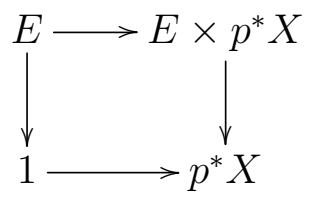

where the map $E \rightarrow p^{*} X$ is the transpose of $p_{!} E \rightarrow X$. This square is a pullback.

In this situation, we have an adjoint equivalence over $\mathscr{F}$ :

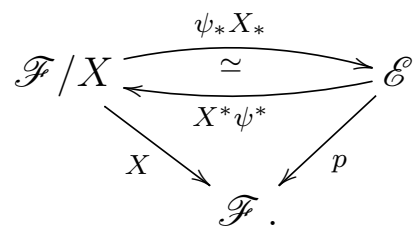

In fact, this equivalence extends to slices in the following sense. 
Lemma 2.2. If $\psi: \mathscr{F} \longrightarrow \mathscr{E}$ has an étale section $p$, then for each object $E$ of $\mathscr{E}$, we have an equivalence $\mathscr{F} / p_{!} E \simeq \mathscr{E} / E$ over $\mathscr{F} / \psi^{*} E$.

Proof. This is a straightforward consequence of the case $E=1$ by considering the following diagram.

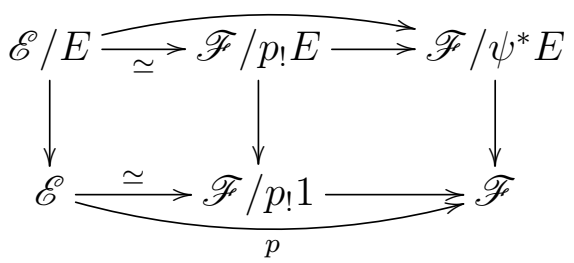

The right hand square a pullback because $p_{!} E \cong \psi^{*} E \times p_{!}(1)$. We also have $\mathscr{E} / E \simeq$ $\mathscr{E} / p^{*} \psi^{*} E$ because $p^{*} \psi^{*} \cong \mathrm{I}$, so that the outer square is a pullback. Therefore, the left hand square is a pullback, and because the bottom map in it is an equivalence, so is the top one.

For any object $X$ of a topos, $X^{X}$ is a monoid under internal composition, whence so is $\psi_{*}\left(X^{X}\right)$ for any geometric morphism $\psi$.

Lemma 2.3. Let $p$ be an étale section of a locally connected $\psi: \mathscr{F} \longrightarrow \mathscr{E}$. For $X=p_{!}(1)$ and $G=\psi_{*}\left(X^{X}\right)$ as above we have:

(i) $G \cong \psi_{!}(X \times X)$;

(ii) $G$ carries the structure of a group;

(iii) $G$ acts on every object of the form $p^{*} F$.

Proof. In this case, the adjoint equivalence $X^{*} \psi^{*} \dashv \psi_{*} X_{*}$ has a left adjoint $\psi_{!} \Sigma_{X}$, which must be isomorphic to $\psi_{*} X_{*}$. Therefore, for any object $F$ of $\mathscr{F}$ we have:

$$
p^{*} F \cong \psi_{*}\left(F^{X}\right) \cong \psi_{*}\left(X_{*} X^{*} F\right) \cong \psi_{!} X_{!} X^{*} F \cong \psi_{!}(X \times F) .
$$

In particular, taking $F=X$, we obtain $G=\psi_{*}\left(X^{X}\right) \cong \psi_{!}(X \times X)$.

For (ii) note that the twist map on $X \times X$ induces an involution on $G$, and that this is actually the inverse for the monoid operation on $G$. Details are left to the reader.

For (iii) observe that the monoid $X^{X}$ acts canonically on objects of the form $F^{X}$ via

$$
F^{X} \times X^{X} \rightarrow X^{X} ; \quad(m, a) \mapsto m a
$$

This action passes via $\psi_{*}$ to one of $G=\psi_{*}\left(X^{X}\right)$ on objects $\psi_{*}\left(F^{X}\right) \cong p^{*} F(5)$ as required.

We are ready for the result we need. 
Theorem 2.4. A geometric morphism with codomain $\mathscr{E}$ is connected atomic with an étale section if and only if it is equivalent to

$$
\mathcal{B}(\mathscr{E} ; G) \longrightarrow \mathscr{E}
$$

where $G$ is a group internal to $\mathscr{E}$. In this situation, the étale section is necessarily a surjection.

Proof. One direction is established in $\S 2.1$. For the other direction, we first use Lemma 2.3 to extract the group $G=\psi_{*}\left(X^{X}\right)$ in $\mathscr{E}$. In order to show that $\mathscr{F} \simeq \mathcal{B}(\mathscr{E} ; G)$ consider the following diagram of inverse image functors.

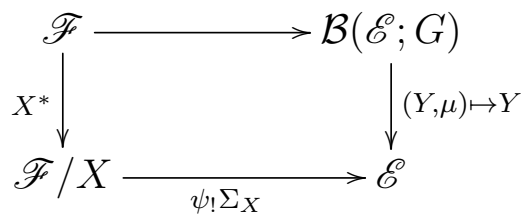

The top horizontal map sends an object $F$ of $\mathscr{F}$ to $p^{*} F$ equipped with the canonical action by $G$ as explained in Lemma 2.3. The diagram commutes because for each $F$ we have

$$
\psi_{!} \Sigma_{X} X^{*}(F)=\psi_{!}(X \times F) \cong p^{*} F .
$$

To prove that the top map is an equivalence observe that both vertical maps are monadic: on $\mathscr{F} / X$ we have the monad $T=X^{*} \Sigma_{X}$, and on $\mathscr{E}$ we have the monad $S=-\times G$. It thus suffices to show that the bottom horizontal equivalence $\psi_{!} \Sigma_{X}$ is a morphism of monads. It is a morphism of endofunctors because we have $\left(\psi_{!} \Sigma_{X}\right) T\left(X^{*} \psi^{*}\right) \cong S$. Indeed, for any $E$ in $\mathscr{E}$, we have:

$$
\begin{aligned}
& \left(\psi_{!} \Sigma_{X}\right) T\left(X^{*} \psi^{*}\right)(E)=\left(\psi_{!} \Sigma_{X}\right)\left(X^{*} \Sigma_{X}\right)\left(X^{*} \psi^{*} E\right) \\
\cong & \psi_{!}\left(\psi^{*} E \times X \times X\right) \cong E \times \psi_{!}(X \times X) \cong E \times G .
\end{aligned}
$$

It is readily seen that this isomorphism respects the monad structures because the monad structure on $-\times G$ is defined in terms of the diagonal and projections of $X$.

\subsection{Locally split geometric morphisms}

In our study of locally anisotropic toposes it turns out that we must consider geometric morphisms that, although they may not be split, still satisfy the weaker condition of being locally split.

Definition 2.5. A local section of a geometric morphism $\psi: \mathscr{F} \longrightarrow \mathscr{E}$ consists of a globally supported object $E$ of $\mathscr{E}$ and a geometric morphism $p$ making the following diagram commute:

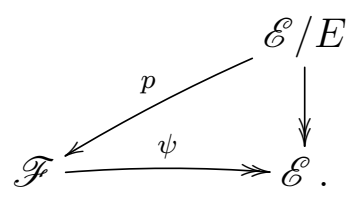


A geometric morphism with a local section is necessarily a surjection. Moreover, a local section $p$ of $\psi$ can alternatively be described by a genuine section $\bar{p}$ of the morphism $\bar{\psi}$ arising in the following topos pullback.

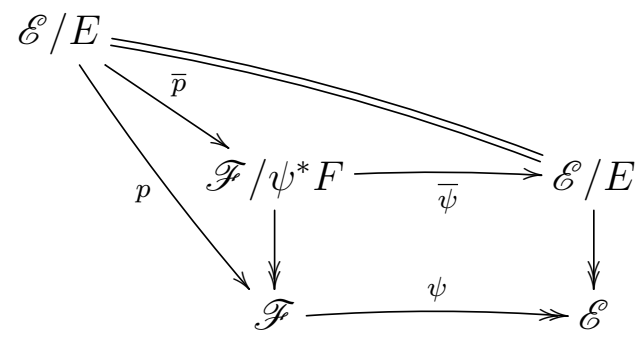

We sometimes refer to a geometric morphism with a local section as locally split.

\section{Basic isotropy theory}

The purpose of the present section is to provide explicit descriptions of the behaviour of the isotropy invariant under two typical constructions: the formation of slice toposes and of toposes of group actions. The important case of slicing by the isotropy group itself gives what we call the topos of crossed sheaves. We also provide a calculation of the isotropy group of $\mathcal{B}(\mathscr{E} ; G)$ as a semidirect product that we need in subsequent argumentation.

\subsection{Definition of the isotropy group}

The introduction explains that the isotropy group $Z$ of a topos $\mathscr{E}$ classifies internal symmetry in the sense that morphisms $X \rightarrow Z$ correspond to automorphisms of the étale geometric morphism

$$
\Sigma_{X} \dashv X^{*} \dashv X_{*}: \mathscr{E} / X \longrightarrow \mathscr{E}
$$

associated with $X$. Such an automorphism may be regarded as an automorphism of the left adjoint $\Sigma_{X}$ : this amounts to a compatible family $Y \stackrel{t_{f}}{\rightarrow} Y$ of isomorphisms of $\mathscr{E}$, indexed by $Y \stackrel{f}{\rightarrow} X$. Compatibility means that for any composable pair $Y^{\prime} \stackrel{g}{\rightarrow} Y \stackrel{f}{\rightarrow} X$, the square

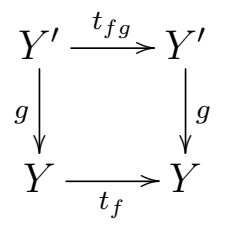

commutes. In particular, for every $Y \stackrel{f}{\rightarrow} X$ we have a commutative diagram

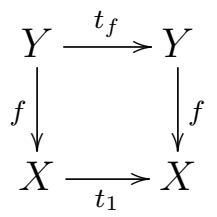


Intuitively, an element $X \stackrel{t}{\rightarrow} Z$ of isotropy encodes a way to formally conjugate the automorphism $t_{1}$ of $X$ by maps $Y \stackrel{f}{\rightarrow} X$. Indeed, if $f$ were invertible, then we must have $t_{f}=f^{-1} t_{1} f$.

Equivalently, we may regard an element of isotropy $X \stackrel{t}{\rightarrow} Z$ as an automorphism of the inverse image functor $X^{*}$ : this amounts to an 'action'

$$
Y \times X \rightarrow Y ;(y, x) \mapsto y x
$$

for every object $Y$ of $\mathscr{E}$, such that its pairing $Y \times X \rightarrow Y \times X$ with the projection to $X$ is an isomorphism.

In particular, the identity map $1_{Z}$ corresponds to an automorphism of $\mathscr{E} / Z \longrightarrow \mathscr{E}$, which we denote $\theta$. It follows that

(i) $\theta_{Y}: Y \times Z \rightarrow Y$ is a group action, and

(ii) $\theta_{Z}(w, z)=z^{-1} w z$.

We refer to $\theta$ as the universal action of $Z$.

Example 3.1. The isotropy group of $\mathcal{B}($ Set; $G)$ is $\bar{G}$, the group $G$ equipped with the conjugation action. Its action on an object $(X, \mu)$ of $\mathscr{E}$ is given by $\mu:(X, \mu) \times \bar{G} \rightarrow(X, \mu)$.

A localic topos is anisotropic, but the class of anisotropic toposes is significantly more inclusive than the localic ones. For example, the topos of presheaves on a small category that is rigid, in the sense of having no non-trivial automorphisms, is anisotropic. A subtopos of an anisotropic topos may not be anisotropic; however, an open subtopos always is. More generally, a slice topos of an anisotropic topos is anisotropic ( $§ 3.2)$.

We end this section with a brief recapitulation of how to transport isotropy across a locally connected geometric morphism. Whiskering an automorphism of $\mathscr{F} / X \longrightarrow \mathscr{F}$ with a locally connected morphism $\psi: \mathscr{F} \longrightarrow \mathscr{E}$ factors via $\mathscr{F} / X \longrightarrow \mathscr{E} / \psi_{!}(X)$ through an automorphism of $\mathscr{E} / \psi_{!}(X) \longrightarrow \mathscr{E}$. Thus, a morphism $X \rightarrow Z_{\mathscr{F}}$ passes to a morphism $\psi_{!}(X) \rightarrow Z_{\mathscr{E}}$, whence to one $X \rightarrow \psi^{*}\left(Z_{\mathscr{E}}\right)$. Ultimately, this gives a map

$$
m_{\psi}=m: Z_{\mathscr{F}} \rightarrow \psi^{*}\left(Z_{\mathscr{E}}\right)
$$

in $\mathscr{F}$, which we refer to as the comparison homomorphism associated with $\psi$. We record the following straightforward fact for future reference.

Lemma 3.2. If $\phi: \mathscr{G} \longrightarrow \mathscr{F}$ and $\psi: \mathscr{F} \longrightarrow \mathscr{E}$ are locally connected, then the associated comparison morphisms form a commutative diagram.

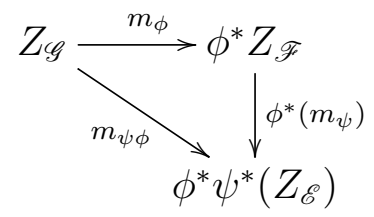




\subsection{Isotropy group of $\mathscr{E} / X$}

The isotropy group of a slice topos is a natural construction. For instance, in the topos $\mathcal{B}($ Set; $G) / X$ it is the bundle of stabilizers $\coprod \operatorname{Stab}_{x} \rightarrow X$ of a $G$-set $X$. Basically the same idea works in general.

Let $X$ denote an arbitrary object of a topos $\mathscr{E}$. As usual, $\theta_{X}$ denotes the universal action by the isotropy group of $\mathscr{E}$ on $X$. Let $\zeta_{X}: Z(X) \rightarrow X$ denote the isotropy group of $\mathscr{E} / X$.

Proposition 3.3. The isotropy group $\zeta_{X}$ of $\mathscr{E} / X$ is the equalizer in $\mathscr{E}$

$$
Z(X) \longrightarrow X \times Z \stackrel{\theta_{X}}{\longrightarrow} X
$$

of the universal action $\theta_{X}$ and the projection. In particular, $\zeta_{X}$ is a subgroup of $X^{*}(Z)$.

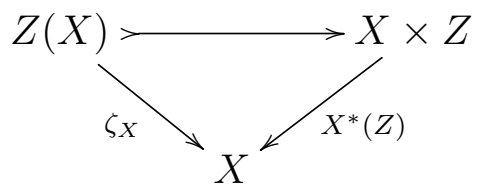

Proof. To show that $\zeta_{X}$ does indeed classifies isotropy in $\mathscr{E} / X$, consider an object $Y \stackrel{f}{\rightarrow} X$ of $\mathscr{E} / X$. A morphism

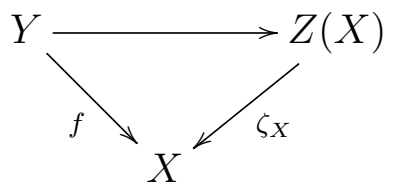

corresponds, using composition with $Z(X) \rightarrow X \times Z \rightarrow Z$, to an element of isotropy $Y \stackrel{t}{\rightarrow} Z$ in $\mathscr{E}$ such that

$$
f\left(t_{Y}(y)\right)=f(y t(y))=f(y) t(y)=f(y)
$$

for all $y \in Y$. That is, $t$ is an element of isotropy of $Y$ that is annihilated by $f$. This says that $t$ may be regarded as an automorphism of

$$
f: \mathscr{E} / Y \longrightarrow \mathscr{E} / X \text {. }
$$

It is easily verified that this correspondence between morphisms $f \rightarrow \zeta_{X}$ and automorphisms of $f: \mathscr{E} / Y \longrightarrow \mathscr{E} / X$ is a bijective one.

In element-style notation, we have $Z(X)=\{(x, z) \mid x z=x\}$. Hence we may regard $\zeta_{X}$ as the bundle of stabilizer subgroups:

$$
\begin{gathered}
\coprod_{x \in X} \operatorname{Stab}(x) \\
\sqrt{\downarrow} \\
X
\end{gathered}
$$


where $\operatorname{Stab}(x)=\{z \in Z \mid x z=x\}$.

The universal action of $\zeta_{X}$ on an arbitrary object $Y \stackrel{f}{\rightarrow} X$ is a map $\theta_{f}$ over $X$ as in the following diagram.

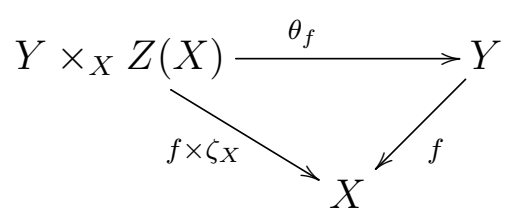

We have

$$
Y \times_{X} Z(X)=\{(y, z) \in Y \times Z \mid f(y) z=f(y)\}
$$

and

$$
\theta_{f}(y, z)=\theta_{Y}(y, z)=y z
$$

Observe that (9) does indeed commute because $f(y z)=f(y) z=f(y)$.

Lemma 3.4. For any object $X$ of $\mathscr{E}$, the isotropy group $\zeta_{X}$ of $\mathscr{E} / X$ acts trivially on an object $Y \stackrel{t}{\rightarrow} X$ of $\mathscr{E} / X$ if and only if

$$
\forall y \in Y, z \in Z: t(y) z=t(y) \Rightarrow y z=y .
$$

Definition 3.5. An object $X$ of a topos $\mathscr{E}$ is said to be anisotropic if the slice topos $\mathscr{E} / X$ is anisotropic: $\zeta_{X}=1_{X}$. Thus, $X$ is anisotropic if and only if

$$
\forall z \in Z, x \in X(x z=x \Rightarrow z=u)
$$

where $u$ denotes the unit of $Z$.

The following is our main definition.

Definition 3.6. We shall say that a topos is locally anisotropic if it has a globally supported anisotropic object.

Thus, a topos is locally anisotropic if it admits an étale covering by an anisotropic topos. By way of comparison, an étendue is a topos that is locally a localic topos. Since the class of anisotropic toposes is wider than that of localic toposes, the class of locally anisotropic toposes comprises all étendues, but is again wider.

\subsection{Crossed sheaves}

So far we have regarded morphisms $X \rightarrow Z$ as generalized elements of $Z$, corresponding to elements of isotropy of $X$. However, we may equally well regard a morphism $X \rightarrow Z$ as an object of the slice topos $\mathscr{E} / Z$. As such, we sometimes refer to $X \stackrel{t}{\rightarrow} Z$ as a crossed sheaf on $\mathscr{E}$, and to $\mathscr{E} / Z$ as the topos of crossed sheaves on $\mathscr{E}$. Sometimes it is convenient to denote a crossed sheaf on $\mathscr{E}$ by $t: X \longrightarrow \mathscr{E}$. 
Remark 3.7. Freyd and Yetter [2] consider crossed sheaves in the special case $\mathscr{E}=$ $\mathcal{B}($ Set $; G)$, the topos of right $G$-sets for a discrete group $G$, calling them crossed $G$ sets. We have adopted this terminology with the understanding that we are conflating the notion of sheaf on a site for $\mathscr{E}$ with that of sheaf on $\mathscr{E}$ because we may regard $\mathscr{E}$ as a canonical site for itself. We return to crossed sheaves in our concluding discussion $(\S 6)$.

A morphism of crossed sheaves

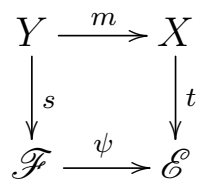

consists of a geometric morphism $\psi$ and a map $Y \stackrel{m}{\rightarrow} \psi^{*} X$ in $\mathscr{F}$ such that for every object $E$ of $\mathscr{E}$ the diagram below commutes.

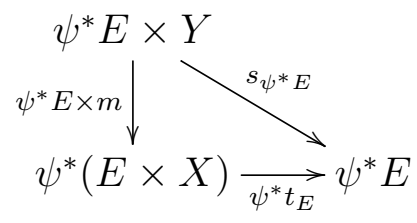

This diagram may be factored as the following one

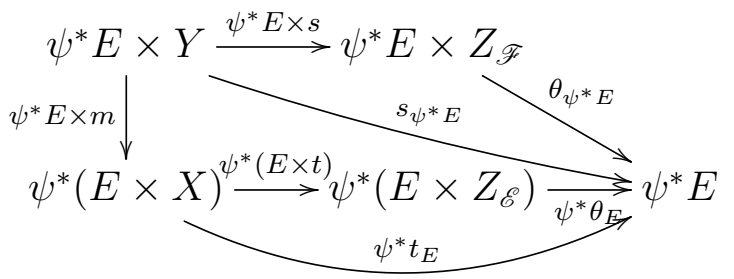

so that $(\psi, m)$ is a morphism of crossed sheaves if and only if

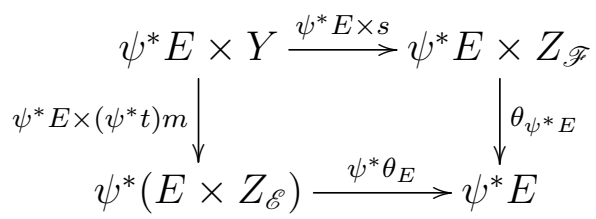

commutes for every object $E$ of $\mathscr{E}$. We may also write $\psi s=t m$ to indicate that $(\psi, m)$ is a morphism of crossed sheaves. The following whiskering diagram is yet another way to think of a morphism of crossed sheaves.

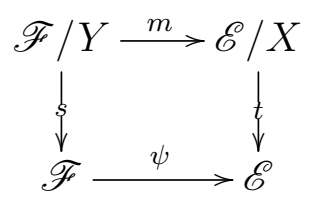


Here we regard $m$ as a geometric morphism whose inverse image $m^{*} p$ is given by the following pullback.

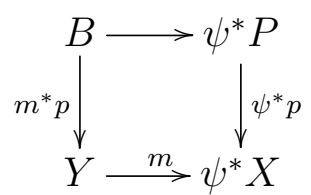

The pair $(\psi, m)$ is said to be Cartesian when $m$ is an isomorphism, and vertical when $\psi$ is the identity geometric morphism.

Remark 3.8. If $G$ is a group internal to a topos $\mathscr{E}$, then a group homomorphism $G \stackrel{\delta}{\rightarrow} Z$ is a special kind of crossed sheaf. The universal action of $Z$ on $G$ makes $G$ a right $Z$-module and $\delta$ a $Z$-equivariant map. If $\delta$ also satisfies the Peiffer identity $g \delta(h)=h^{-1} g h$, then $\delta$ is a crossed $Z$-module. Such a structure is equivalent to a crossed topos on $\mathscr{E}$ ([5], Corollary 5.7). A morphism $(\psi, m)$ of crossed toposes is the same as for crossed sheaves, except that $m$ is required to be a group homomorphism.

Let us denote the isotropy group of the topos of crossed sheaves $\mathscr{E} / Z$ by

$$
\zeta_{Z}=\zeta: Z(Z) \rightarrow Z
$$

By $\S 3.2$ we find that it is the subgroup of $Z^{*}(Z)$ consisting of all pairs $(w, z)$ such that $z w=w z$, i.e., of all commuting pairs.

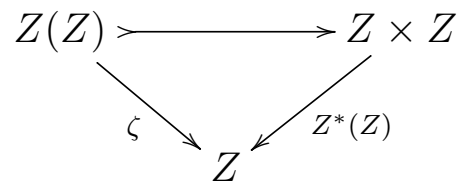

The multiplication in $\zeta$ is fiberwise over $Z$ :

$$
(w, z)(w, v)=(w, z v)
$$

which is again a commuting pair. We may also regard $\zeta$ as the bundle of commutator subgroups

$$
\coprod_{z \in Z} C(z) \rightarrow Z
$$

where $C(z)$ denotes the subgroup $\{w \mid w z=z w\}$ of $Z$.

The universal action of $\zeta$ on a crossed sheaf $X \stackrel{t}{\rightarrow} Z$ is then a map $\theta_{t}$ over $Z$

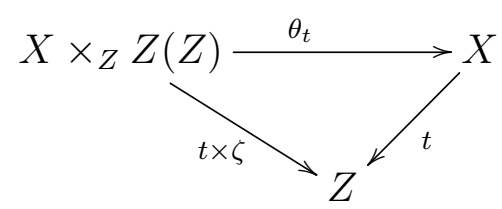


where

$$
X \times{ }_{Z} Z(Z)=\{(x, w) \in X \times Z \mid w t(x)=t(x) w\} .
$$

Explicitly, the action is given by the formula

$$
\theta_{t}(x, w)=\theta_{X}(x, w)=x w .
$$

\subsection{Isotropy group of $\mathcal{B}(\mathscr{E} ; G)$}

We calculate the isotropy group of $\mathcal{B}(\mathscr{E} ; G)$, the topos of right $G$-objects in $\mathscr{E}$, where $G$ is a group internal to $\mathscr{E}$. As in $\S 2.1$ we typically denote objects of this topos by $(X, \mu)$, often writing the action as $\mu(x, g)=x g$. We denote the étale section of the structure morphism (3) by $p$, corresponding to the unit $1 \stackrel{u}{\rightarrow} G$.

In preparation for the calculation, observe that morphisms $p_{!} A \rightarrow p_{!} B$ in $\mathcal{B}(\mathscr{E} ; G)$ correspond by adjointness to morphisms

$$
A \rightarrow p^{*} p_{!}(B) \cong p^{*}\left(\psi^{*} B \times G\right) \cong B \times G
$$

in $\mathscr{E}$. The unit of the adjointness is the map $A \stackrel{\left(1_{A}, u\right)}{\rightarrow} A \times G$, and composition of morphisms $A \stackrel{(\alpha, g)}{\rightarrow} B \times G$ and $B \stackrel{(\beta, h)}{\rightarrow} C \times G$ is given by

$$
(\beta, h) \circ(\alpha, g)(a)=(\beta \alpha(a), h(\alpha(a)) \cdot g(a)) .
$$

This is simply the Kleisli composition for the monad defining $\mathcal{B}(\mathscr{E} ; G)$ :

$$
A \stackrel{(\alpha, g)}{\longrightarrow} B \times G \stackrel{(\beta, h) \times G}{\longrightarrow} C \times G \times G \stackrel{C \times \text { mult }}{\longrightarrow} C \times G .
$$

It follows right away that a morphism $p_{!} A \rightarrow p_{!} B$ is an isomorphism if and only if the corresponding $A \stackrel{(\alpha, g)}{\rightarrow} B \times G$ has the property that $\alpha$ is an isomorphism: there are no restrictions on $g$ for this to happen.

Let us denote the isotropy group of $\mathcal{B}(\mathscr{E} ; G)$ by $Z_{G}$. As always, $Z$ is the isotropy group of $\mathscr{E}$. We shall first determine the underlying object of $Z_{G}$, which is $p^{*}\left(Z_{G}\right)$. An element $E \stackrel{t}{\rightarrow} p^{*} Z_{G}$ corresponds to a morphism $p_{!} E \rightarrow Z_{G}$. Because $Z_{G}$ classifies isotropy of $\mathcal{B}(\mathscr{E} ; G)$, such a morphism corresponds to an automorphism of the map

$$
\mathcal{B}(\mathscr{E} ; G) / p_{!} E \longrightarrow \mathcal{B}(\mathscr{E} ; G)
$$

However, by the canonical equivalence $\mathcal{B}(\mathscr{E} ; G) / p_{!} E \simeq \mathscr{E} / E$ (Lemma 2.2), $t$ may be regarded as an automorphism of the geometric morphism $\mathscr{E} / E \rightarrow \mathcal{B}(\mathscr{E} ; G)$. The leftmost adjoint of this morphism is given by

$$
\mathscr{E} / E \longrightarrow \mathcal{B}(\mathscr{E} ; G) ; \quad X \stackrel{f}{\rightarrow} E \longmapsto p_{!} X=\psi^{*} X \times G .
$$


As such, an element of isotropy $t$ supplies for every $X \stackrel{f}{\rightarrow} E$ an automorphism $p_{!} X \stackrel{t_{f}}{\rightarrow} p_{!} X$ in $\mathcal{B}(\mathscr{E} ; G)$, which is natural in $f$. According to our description of morphisms $p_{!} X \rightarrow p_{!} X$ above, the component $t_{f}$ may be specified as a map $t_{f}=\left(\alpha_{f}, g_{f}\right): X \rightarrow X \times G$ in $\mathscr{E}$. By the naturality of $t$, the diagram

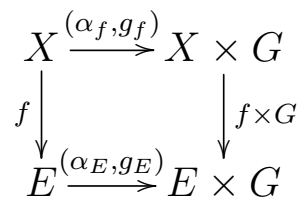

commutes. Because each component $t_{f}$ is an isomorphism, so is each $\alpha_{f}$. This means that the components $\alpha_{f}$ form an element of isotropy $E \stackrel{\alpha}{\rightarrow} Z$, and that $g_{f}=g_{E} f$, and hence that the family $g_{f}$ is determined solely by $g=g_{E}$. We have thus shown that elements $E \stackrel{t}{\rightarrow} p^{*} Z_{G}$ are in one-one correspondence with pairs $(\alpha, g)$. We conclude that $p^{*} Z_{G} \cong Z \times G$ : the underlying object of $Z_{G}$ is $Z \times G$.

Theorem 3.9. The isotropy group $Z_{G}$ of $\mathcal{B}(\mathscr{E} ; G)$ and its universal action are characterized by the following:

(i) the underlying group $p^{*} Z_{G}$ of $Z_{G}$ is the semidirect product $Z \ltimes G$ arising from the universal action of $Z$ on $G$. Explicitly, the multiplication and inverse are given by

$$
(z, g)(w, h)=(z w,(g w) h) ; \quad(z, g)^{-1}=\left(z^{-1}, g^{-1} z^{-1}\right) .
$$

(ii) the universal action of $Z_{G}$ on an object $(X, \mu)$ of $\mathcal{B}(\mathscr{E} ; G)$ is given by

$$
x(z, g)=\mu(x z, g)=(x z) g,
$$

where $x z$ is the universal action of $Z$ on $G$;

(iii) the action of $G$ on $Z_{G}$ is given by

$$
(z, g) h=\left(z,(h z)^{-1} h g\right) .
$$

Proof. (i) We have already established that the underlying object of $Z_{G}$ must be $Z \times G$. Since the multiplication in $Z_{G}$ corresponds to composition of isotropy automorphisms, and since under the adjointness $p_{!} \dashv p^{*}$ this composition corresponds to the Kleisli composition, we see that the multiplication on $Z \times G$ is the semidirect one, arising from the canonical action of $Z$ on $G$.

(ii) Since every isotropy element of $(X, \mu)$ in $\mathcal{B}(\mathscr{E} ; G)$ has an underlying isotropy element of $X$ in $\mathscr{E}$, we know that $x(z, 1)$ must equal $x z$. On the other hand, the action by elements of the form $(1, g)$ has to be $x(1, g)=\mu(x, g)=x g$. 
(iii) The action of $G$ on the first component $Z$ of $Z \ltimes G$ must be trivial; this follows right away from the fact that the group homomorphism $Z_{G} \rightarrow \gamma^{*} Z$, which is simply the projection $Z \ltimes G \rightarrow Z$, must be $G$-equivariant; since the $G$-action on $\gamma^{*} Z$ is trivial, the action of $G$ on the first component of $Z_{G}$ must be trivial as well. Thus the action has the form

$$
(z, g) \cdot h=(z, a)
$$

where $a$ is to be determined. To do so consider the $Z_{G}$-action on Yoneda $G$ :

$$
G \times Z_{G} \rightarrow G
$$

given by $h(z, g)=(h z) g$. In particular, we have $u(z, g)=g$, where $u$ is the unit of $G$. By the $G$-equivariance of the action of $Z_{G}$ on $G$, we have

$$
g h=(u(z, g)) \cdot h=(u \cdot h)((z, g) \cdot h)=h(z, a)=(h z) a
$$

so that $a=(h z)^{-1} g h$. It follows from the fact that multiplication in $G$ is $Z$-equivariant that this action by $G$ on $Z_{G}$ is associative.

Remark 3.10. We may gain another perspective on Theorem 3.9 as follows. The kernel $Z_{\psi}$ of the comparison map $Z_{\mathscr{F}} \rightarrow \psi^{*} Z_{\mathscr{E}}(8)$ associated with a geometric morphism $\psi:$ $\mathscr{F} \longrightarrow \mathscr{E}$ can be considered as the isotropy group of $\mathscr{F}$ relative to $\psi$. An element of relative isotropy $X \stackrel{t}{\rightarrow} Z_{\psi}$ is simply one $X \rightarrow Z_{\mathscr{F}}$ of $\mathscr{F}$ corresponding to an automorphism of $\mathscr{F} / X \longrightarrow \mathscr{F}$ that is annihilated by $\psi$ in the sense that the whiskering

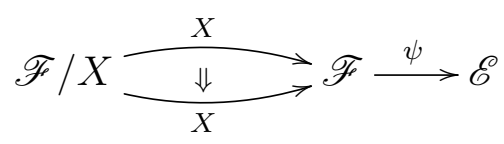

equals the identity. For instance, the relative isotropy group of (3) must have $G$ as its underlying group: $p^{*} Z_{\gamma} \cong G$. Now consider the following sequence of groups in $\mathcal{B}(\mathscr{E} ; G)$ :

$$
1 \rightarrow Z_{\gamma} \rightarrow Z_{G} \rightarrow \gamma^{*} Z \rightarrow 1
$$

to which we can apply the underlying object functor $p^{*}$ giving

$$
1 \rightarrow G \rightarrow p^{*} Z_{G} \rightarrow Z \rightarrow 1
$$

This sequence is split by the comparison homomorphism $Z \stackrel{m}{\rightarrow} p^{*} Z_{G}$ of $p$ (8), exhibiting the underlying group of $Z_{G}$ as the semidirect product of $Z$ with $G$ (using Lemma 3.2). Because $p^{*}$ reflects epimorphisms it follows that (14) is exact as well; however, (14) is not necessarily split because the section $m$ is generally not $G$-equivariant. 


\section{Isotropy torsors and the structure theorem}

We turn to one of the central constructions of isotropy theory: the isotropy quotient of a topos [5]. We begin by establishing the "fundamental lemma" of isotropy as we call it, stating that the isotropy quotient of a topos commutes with slicing. Our development of the main theorem (Thm. 4.16) relies on the notion of isotropy torsor and its equivalence with étale splittings of the isotropy quotient. Subsequently, we generalize this to toposes for which the isotropy quotient is locally split by an étale section. Finally, we relate this result to isotropy algebras: these are equivalently given as discrete fibrations on the topos category in our former article [5].

\subsection{Fundamental lemma}

We are mostly interested in the terminal isotropy quotient of a topos (15), but it is worthwhile to observe that the construction is parametrized by crossed sheaves. In fact, the coequifier diagram

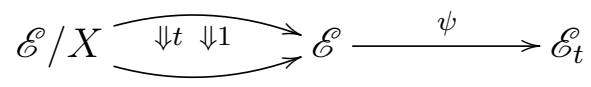

associated with a crossed sheaf $X \stackrel{t}{\rightarrow} Z$ is a topos we call the isotropy quotient of $t$. The category $\mathscr{E}_{t}$ is the full subcategory of $\mathscr{E}$ on those objects $E$ for which the action

$$
E \times X \rightarrow E ; \quad(e, x) \mapsto e t(x)
$$

is trivial: $\forall e, x:$ et $(x)=e$. The inverse image $\psi^{*}$ is simply the inclusion of $\mathscr{E}_{t}$ in $\mathscr{E}$. Its left adjoint $\psi_{\text {! }}$ is the orbit space of the universal action by $Z$ : this is the coequalizer

$$
E \times X \stackrel{(e, x) \mapsto e t(x)}{\stackrel{(e, x) \mapsto e}{\longrightarrow}} E \stackrel{\text { unit }}{\longrightarrow} \psi_{!} E .
$$

The equalizer of the exponential transposes of these maps gives the right adjoint $\psi_{*}$ :

$$
\psi_{*} E \stackrel{\text { counit }}{\longrightarrow} E \longrightarrow E^{X} \text {. }
$$

It follows that $\mathscr{E}_{t}$ is a topos, and that $\psi$ is a connected atomic geometric morphism.

As always, $1 \stackrel{u}{\rightarrow} Z$ denotes the unit of $Z$.

Proposition 4.1. A crossed sheaf $X \stackrel{t}{\rightarrow} Z$ is trivial in the sense that $t(x)=u$ for all $x \in X$ if and only if the isotropy quotient $\mathscr{E} \longrightarrow \mathscr{E}_{t}$ associated with $t$ is an equivalence.

Proof. The isotropy quotient $\mathscr{E} \longrightarrow \mathscr{E}_{t}$ is an equivalence precisely when the automorphism of $\mathscr{E} / X \longrightarrow \mathscr{E}$ corresponding to $t$ equals the identity. This is the case if and only if for any object $E$, we have $e t(x)=e$. However, the morphism $X \rightarrow 1 \rightarrow Z$ also has this property, so that $t$ equals this morphism if and only if the isotropy quotient of $t$ is an equivalence. 

by

We denote the isotropy quotient associated with the terminal crossed sheaf $\theta: Z \longrightarrow \mathscr{E}$

$$
\psi: \mathscr{E} \longrightarrow \mathscr{E}_{\theta}
$$

The topos $\mathscr{E}_{\theta}$ consists of all isotropically trivial objects: those for which the universal action by the isotropy group is trivial.

Proposition 4.2. The following conditions are equivalent for a topos $\mathscr{E}$ :

(i) $\mathscr{E}$ is anisotropic;

(ii) the isotropy quotient of $\mathscr{E}$ is an equivalence;

(iii) every object of $\mathscr{E}$ is isotropically trivial;

(iv) every object of $\mathscr{E}$ is anisotropic;

(v) $\mathscr{E}$ has a globally supported isotropically trivial, anisotropic object.

Proof. If $\mathscr{E}$ is anisotropic, then easily conditions (ii) through (v) hold. On the other hand, (ii) $\Rightarrow$ (i) is a consequence of Prop. 4.1, (ii) and (iii) are equivalent, and (iv) obviously implies (i). Condition (v) implies that $\mathscr{E}$ is anisotropic because if $X$ is an isotropically trivial object of $\mathscr{E}$, then the isotropy group of $\mathscr{E} / X$ is $X \times Z \rightarrow X$. If $X$ is anisotropic, then this group is the trivial one $X \rightarrow X$. If $X$ is globally supported, then $Z=1$.

By comparing inverse image functors it follows that the isotropy quotients of $\mathscr{E}$ and $\mathscr{E} / X$ form a commutative square, as illustrated in the following diagram.

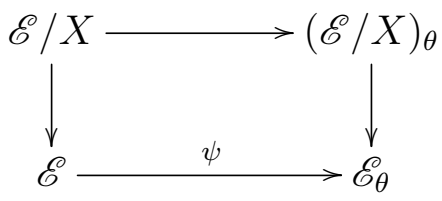

Lemma 4.3 (The fundamental lemma of isotropy). Let $X$ be an object of a topos $\mathscr{E}$, and let $O_{X}=\psi_{1} X$ denote the orbit object of the universal action $\theta_{X}$. Then $(\mathscr{E} / X)_{\theta} \simeq \mathscr{E}_{\theta} / O_{X}$ : the factorization (16) of the composite $\mathscr{E} / X \longrightarrow \mathscr{E} \longrightarrow \mathscr{E}_{\theta}$, and its connected atomic, étale factorization are equivalent.
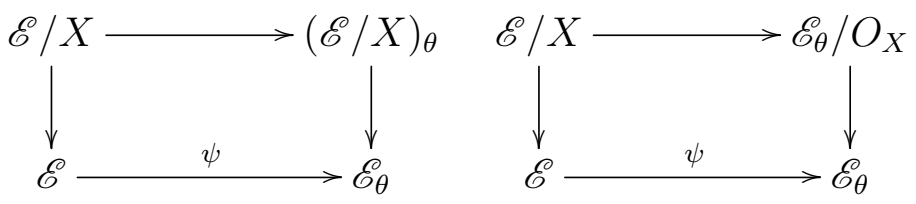
Proof. The equivalence associates with $Y \stackrel{t}{\rightarrow} X$ of $(\mathscr{E} / X)_{\theta}$ the map of orbit spaces $O_{Y} \rightarrow O_{X}$ induced by $t$, as an object of $\mathscr{E}_{\theta} / O_{X}$. On the other hand, it associates with an object $E \stackrel{p}{\rightarrow} O_{X}$, such that $Z$ acts trivially on $E$, the pullback

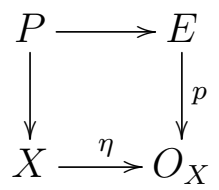

in $\mathscr{E}: \eta$ is the unit of $\psi_{!} \dashv \psi^{*}$. This does indeed give an equivalence. On the one hand, if we start with $p$, then $E \cong O_{P}$ because $\psi$ is locally connected (use Frobenius reciprocity for $\left.\psi_{!} \dashv \psi^{*}\right)$. On the other hand, starting with $Y \stackrel{t}{\rightarrow} X$ on which $\zeta_{X}$ acts trivially, we must show that the induced map $\rho: Y \rightarrow X \times \times_{O_{X}} O_{Y}$, given by $\rho(y)=(t(y), o(y))$, is an isomorphism. In order to see that $\rho$ is an epimorphism suppose that $(x, o(y))$ is a member of the pullback: this means that $t(y)$ and $x$ are in the same orbit in $X$, i.e., $\exists z \in Z$ such that $t(y z)=t(y) z=x$. Hence, $\rho(y z)=(t(y z),[y z]=(x,[y])$. To see that $\rho$ is a monomorphism, suppose that $(t(y),[y])=\left(t\left(y^{\prime}\right),\left[y^{\prime}\right]\right)$, so that $t(y)=t\left(y^{\prime}\right)$ and $y^{\prime}=y z$ for some $z$. Then $t(y z)=t\left(y^{\prime}\right)=t(y)$, so that $y z=y$ by Lemma 3.4, whence $y^{\prime}=y$.

Corollary 4.4. The isotropy quotient $\mathscr{E}_{\theta}$ of a topos $\mathscr{E}$ is anisotropic if and only if $(\mathscr{E} / X)_{\theta}$ is anisotropic for every object $X$ of $\mathscr{E}$.

Proof. If a topos $\mathscr{F}$ is anisotropic, then so is $\mathscr{F} / Y$ because $\zeta_{Y}$ is a subgroup of $Y^{*} Z_{\mathscr{F}}$. In other words, a slice topos of an anisotropic topos is anisotropic. Therefore, if $\mathscr{E}_{\theta}$ is anisotropic, then for any object $X$ of $\mathscr{E}$, the slice topos $\mathscr{E}_{\theta} / O_{X} \simeq(\mathscr{E} / X)_{\theta}$ is anisotropic. The converse implication is trivial by taking $X=1$.

Corollary 4.5. If an object $X$ of a topos $\mathscr{E}$ is anisotropic, then its orbit space $O_{X}$ is an anisotropic object of $\mathscr{E}_{\theta}$. Moreover, the adjunction square

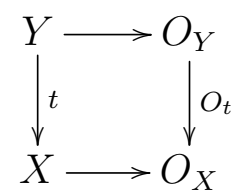

in $\mathscr{E}$ associated with any $t$ is a pullback.

Proof. If $X$ is anisotropic, then

$$
\mathscr{E} / X \simeq(\mathscr{E} / X)_{\theta} \simeq \mathscr{E}_{\theta} / O_{X}
$$

so that $\mathscr{E}_{\theta} / O_{X}$ is anisotropic. Going over and back across this equivalence shows that the adjunction square is a pullback. 
In preparation for our characterization of toposes for which the isotropy quotient has an étale section $(\S 4.2)$ we observe the following.

Lemma 4.6. If the isotropy quotient of a topos $\mathscr{E}$ has a locally connected (hence étale) section, then $\mathscr{E}_{\theta}$ is anisotropic.

Proof. Let $Z_{\theta}$ denote the isotropy group of $\mathscr{E}_{\theta}$. Suppose that $p$ is a locally connected section of $\psi$. We apply Lemma 3.2 to $\psi, p$, and the composite $\psi p \cong 1$ in order to conclude that the diagram below (left)

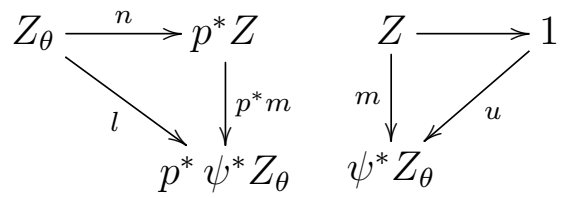

commutes. The isotropy quotient 'kills' $Z$ in the sense that the triangle above (right) commutes. Therefore, $Z_{\theta} \rightarrow 1$ is a monomorphism, whence an isomorphism, because $l$ is an isomorphism.

We also need the following technical result about the isotropy quotient. An object $E$ has associated with it an 'evaluation' map

$$
\xi: Z^{E} \rightarrow E^{E} ; \xi(t)(e)=e t(e)
$$

from a pointwise defined group to an endomorphism monoid. This map may not be a homomorphism in general, but under $\psi_{*}$ it is.

Lemma 4.7. The map $\psi_{*}(\xi)$ is a homomorphism.

Proof. First note that $t \in \psi_{*}\left(Z^{E}\right)$ means that $t \in Z^{E}$ satisfies $t z=t$ for all $z \in Z$. Then

$$
t(e z)=t z(e z)=z^{-1} t(e) z
$$

where the second equality holds because evaluation $Z^{E} \times E \rightarrow Z$ is equivariant, keeping in mind that $Z$ acts on itself by conjugation. Therefore, for $t, s \in \psi_{*}\left(Z^{E}\right)$ we have:

$$
\begin{aligned}
\xi(t) \circ \xi(s)(e) & =\xi(t)(e s(e)) \\
& =(e s(e))(t(e s(e))) \\
& =(e s(e))\left(s(e)^{-1} t(e) s(e)\right) \\
& =e t(e) s(e) \\
& =\xi(t s)(e) .
\end{aligned}
$$




\subsection{Isotropy torsors}

An isotropy torsor is a globally supported object for which the universal action by the isotropy group is free and transitive. In a sense this is opposite to what we call an isotropically trivial object. Indeed, this opposition emerges fully in Prop. 4.11 where we see that isotropy torsors correspond to étale sections of the isotropy quotient.

Definition 4.8. An object $X$ of $\mathscr{E}$ is called an isotropy torsor when it is a $Z$-torsor for the universal action $\theta_{X}$. This means that it must satisfy the following three requirements:

(i) $X$ is globally supported, i.e., the map $X \rightarrow 1$ is an epimorphism;

(ii) the action $\theta_{X}$ is transitive, i.e., we have $\forall x, y \in X(\exists z \in Z x z=y)$; and

(iii) the action $\theta_{X}$ is free, i.e., we have $\forall x \in X \forall z \in Z(x z=z \Rightarrow z=u)$.

We call $X$ connected if it is globally supported and transitive.

As usual, the conditions for a torsor can be formulated diagrammatically: the map

$$
X \times Z \rightarrow X \times X ; \quad(x, z) \mapsto(x, x z)
$$

is injective precisely when $X$ is free, and surjective precisely when $X$ is transitive. An object is anisotropic if and only if it is free. Thus, an object is an isotropy torsor if and only if it is anisotropic and connected if and only if the following diagram is an equalizer and coequalizer.

$$
X \longrightarrow X \times Z^{\stackrel{\theta_{X}}{\longrightarrow}} X \longrightarrow 1
$$

Note that if $O_{X} \cong 1$, then $X$ necessarily has global support.

Remark 4.9. Of course, an isotropy torsor is a $Z$-torsor, but not every $Z$-torsor is an isotropy torsor. For example, the isotropy group $Z$ itself with right multiplication is a $Z$-torsor, but it is only an isotropy torsor when right multiplication coincides with the universal action, i.e., conjugation. This only happens when $Z$ is trivial.

Consider an arbitrary object $X$ of $\mathscr{E}$, and the composite geometric morphism

$$
\mathscr{E} / X \longrightarrow \mathscr{E} \stackrel{\psi}{\longrightarrow} \mathscr{E}_{\theta}
$$

Explicitly, the inverse image functor sends an object $Y$ of $\mathscr{E}_{\theta}$ to the projection $Y \times X \rightarrow X$; its adjoints are given by

$$
E \stackrel{p}{\rightarrow} X \longmapsto \psi_{*} X_{*} p=\left\{y \in X_{*} p \mid \operatorname{Stab}(y)=Z\right\}
$$

and

$$
E \stackrel{p}{\rightarrow} X \longmapsto \psi_{!} \Sigma_{X} p=\psi_{!}(E)=O_{E} .
$$

The composite (17) is atomic, being the composite of an étale map and the atomic $\psi$. 
Proposition 4.10. The composite (17) is

(i) connected if and only if $X$ is connected;

(ii) étale if and only if $X$ is anisotropic;

(iii) an equivalence if and only if $X$ is an isotropy torsor, in which case its two adjoints are isomorphic functors. The quotient $\mathscr{E}_{\theta}$ is anisotropic in this case.

Proof. For (i) note that $X$ is connected if and only if $O_{X} \cong 1$. Thus, the left adjoint of (17) sends the terminal object $1_{X}$ to 1 precisely when $X$ is connected. For (ii) use the Fundamental Lemma 4.3. Statement (iii) holds because a geometric morphism is étale and connected if and only if it is an equivalence.

In order to explain how isotropy torsors are classified by sections of the isotropy quotient consider first an isotropy torsor $X$ in $\mathscr{E}$. We may define a section $p=p_{X}$ of $\psi$

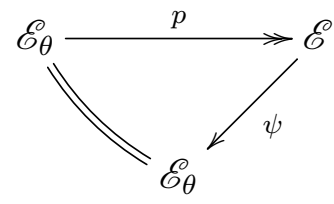

in the following manner: for an object $Y$ of $\mathscr{E}$, let

$$
p^{*}(Y)=\psi_{!}(Y \times X)=O_{Y \times X} .
$$

We may also describe $p^{*}$ by

$$
p^{*}(Y)=\psi_{*}\left(Y^{X}\right) \cong\left\{f \in Y^{X} \mid f \text { is } Z \text {-equivariant }\right\} .
$$

For any object $E$ of $\mathscr{E}_{\theta}$ we have

$$
p_{!}(E)=\psi^{*} E \times X ; p_{*}(E)=\left(\psi^{*} E\right)^{X} .
$$

Thus, the section $p$ obtained from the isotropy torsor $X$ comes from the equivalence of Prop. 4.10:

$$
p: \mathscr{E}_{\theta} \simeq \mathscr{E} / X \longrightarrow \mathscr{E} .
$$

Diagram (18) commutes (up to isomorphism) because

$$
p^{*} \psi^{*}(E)=\psi_{!}\left(\psi^{*} E \times X\right) \cong E \times \psi_{!} X \cong E .
$$

In particular, we find that $p$ is étale.

On the other hand, suppose we are given an étale section $p$ of $\psi$. If $X=p_{!}(1)$, then $\mathscr{E} / \theta \simeq \mathscr{E} / X$. By Lemma 4.6, the quotient $\mathscr{E}_{\theta}$ is anisotropic, so that $X$ is anisotropic. In addition, we have $O_{X}=\psi_{!}(X)=\psi_{!}\left(p_{!}(1)\right) \cong 1$, whence $X$ is connected. Thus, $X$ is an isotropy torsor. 
Proposition 4.11. The above constructions constitute an equivalence between the category of isotropy torsors in $\mathscr{E}$ as a full subcategory of $\mathscr{E}$, and the category of étale sections of $\psi$, whose morphisms are natural transformations over $\mathscr{E}_{\theta}$. These two categories are equivalent groupoids.

Proof. Suppose that $p$ is an étale section of $\psi$. Pairing the transpose $p_{!}(E) \rightarrow \psi^{*} E$ of the isomorphism $\psi_{!} p_{!}(E) \rightarrow$ with $p_{!}(E) \rightarrow p_{!}(1)=X$ yields an isomorphism

$$
p_{!}(E) \rightarrow \psi^{*} E \times X \text {. }
$$

Furthermore, if $p_{!} \stackrel{\phi}{\rightarrow} q_{\text {! }}$ is a natural transformation over $\mathscr{E}_{\theta}$, then for any object $E$ of $\mathscr{E}_{\theta}$, the triangle of isomorphisms below (left) commutes: $\psi_{!}\left(\phi_{E}\right)$ is an isomorphism because the other two morphisms in the triangle are.
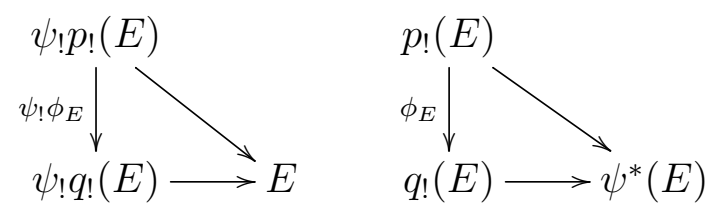

Therefore, the transposed triangle above (right) commutes, so that

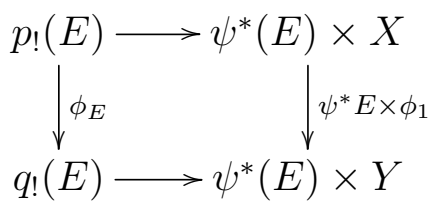

commutes, where $Y=q_{!}(1)$. The horizontal maps are the isomorphisms (20). This establishes the required naturality of the equivalence when passing from étale sections to isotropy torsors and back.

The two categories are indeed groupoids because on the one hand a morphism of torsors in a topos must be an isomorphism. On the other hand, if $p_{!} \stackrel{\phi}{\rightarrow} q_{!}$is over $\mathscr{E}_{\theta}$, then for any $E$, the adjunction square

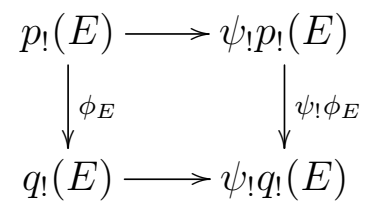

must be a pullback because $q_{!}(E)$ is anisotropic (Cor. 4.5). Therefore, $\phi_{E}$ is an isomorphism because $\psi_{!}\left(\phi_{E}\right)$ is one. We leave the remaining details of the equivalence to the reader.

Theorem 4.12. The following conditions on a topos $\mathscr{E}$ are equivalent: 
(i) $\mathscr{E}$ has an isotropy torsor;

(ii) $\mathscr{E}$ is equivalent to $\mathcal{B}(\mathscr{F} ; G)$, where $G$ is a group internal to an anisotropic topos $\mathscr{F}$ (so that $\mathscr{F}$ is the isotropy quotient of $\mathcal{B}(\mathscr{F} ; G)$ ). In this case, the isotropy quotient $\psi$ has an étale section $p$ (given by the unit $u$ of $G$ ), and the following two diagrams are equivalent.
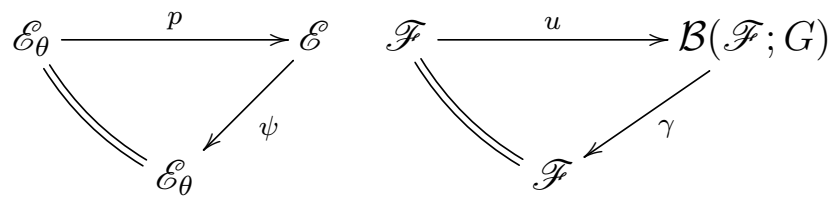

(iii) $\mathscr{E}$ has an object $X$ such that the following two diagrams are equivalent.
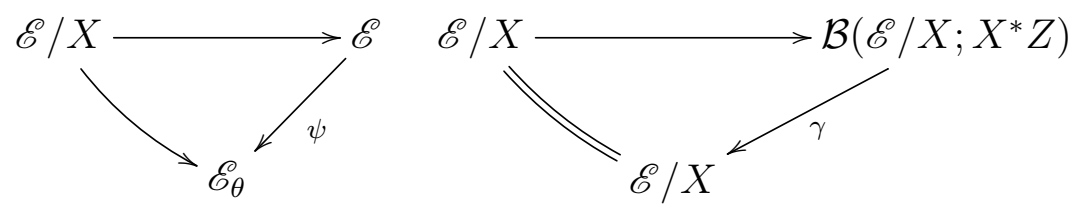

Proof. (i) $\Rightarrow$ (ii). Suppose that $\mathscr{E}$ has an isotropy torsor $X$. By Prop. 4.11, there is corresponding to $X$ an étale section $p$ of the isotropy quotient $\psi$ such that $X \cong p_{!}(1)$. The required anisotropic topos $\mathscr{F}$ is $\mathscr{E}_{\theta} \simeq \mathscr{E} / X$, and the required group $G$ is

$$
p^{*}(X) \cong \psi_{*}\left(X^{X}\right) \cong\left\{f \in X^{X} \mid f \text { is } Z \text {-equivariant }\right\} \text {. }
$$

(ii) $\Rightarrow$ (iii). Suppose that $G$ is a group internal to an anisotropic topos $\mathscr{F}$. By Theorem 3.9, the isotropy group of $\mathcal{B}(\mathscr{F} ; G)$ is $Z_{\mathscr{F}} \ltimes G \cong \bar{G}$ (the latter referring to $G$ with the conjugation action, see Example 3.1). Moreover, $\mathscr{F}$ is the isotropy quotient of $\mathcal{B}(\mathscr{F} ; G)$, Yoneda $G$ is an isotropy torsor of $\mathcal{B}(\mathscr{F} ; G)$, and we have $\mathcal{B}(\mathscr{F} ; G) / G \simeq \mathscr{F}$. Moreover, $G^{*} \bar{G}$ and the group $G$ with which we started are isomorphic.

(iii) $\Rightarrow$ (i). If the two given diagrams are equivalent, then the composite

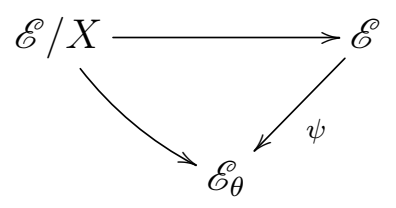

must be an equivalence. Therefore, $X$ is an isotropy torsor.

Corollary 4.13. If $\mathscr{E}$ has an isotropy torsor corresponding to an étale section $p$, then $\mathscr{E}$ is equivalent to $\mathcal{B}\left(\mathscr{E}_{\theta} ; p^{*} Z\right)$, where $\mathscr{E}_{\theta}$ is anisotropic. 
Proof. If $X$ is an isotropy torsor of $\mathscr{E}$, then $X^{X} \cong Z^{X}$. Consequently, the group $G$ in $\mathscr{E}_{\theta}$ for which $\mathscr{E} \simeq \mathcal{B}\left(\mathscr{E}_{\theta} ; G\right)$ is

$$
p^{*}(X) \cong \psi_{*}\left(X^{X}\right) \cong \psi_{*}\left(Z^{X}\right) \cong p^{*}(Z) .
$$

Lemma 4.7 explains that the middle isomorphism is indeed a group homomorphism in $\mathscr{E}_{\theta}$.

Remark 4.14. In our article [5] we explain that isotropy quotients of presheaf toposes can be described purely on the level of the underlying category: each category $\mathscr{C}$ has associated with it a congruence, whose corresponding quotient $\psi: \mathscr{C} \rightarrow \mathscr{C}_{\theta}$ induces the quotient of the topos of presheaves on $\mathscr{C}$. In some cases, the functor $\psi$ has a section $p$, in which case we can use that section to pull back the isotropy group to $\mathscr{C}_{\theta}$. Under certain additional conditions, we may recover $\mathscr{C}$ from $\mathscr{C}_{\theta}$ and $p^{*} Z$ as $\mathscr{C} \cong \mathscr{C}_{\theta} \ltimes p^{*} Z$. This is at the same time more general than Theorem 4.12 and more restrictive: it is more general because the conditions on $\mathscr{C}$ do not preclude $\mathscr{C}_{\theta}$ from being anisotropic, and the section $p$ does not have to induce an étale section. More honestly, we do not know whether a section of $\mathscr{C} \longrightarrow \mathscr{C}_{\theta}$ necessarily induces an étale geometric morphism. It is more restrictive because the additional conditions do not seem to be a consequence of $\mathscr{C}_{\theta}$ being anisotropic.

\subsection{Structure of locally anisotropic toposes}

A topos, while not possessing an isotropy torsor, may still have a globally supported anisotropic object. It is therefore worthwhile to generalize our work in $\S 4.2$ for isotropy torsors and splittings of the isotropy quotients to anisotropic objects and local splittings $(\S 2.3)$. This generalization is almost immediate, but it depends on the Fundamental Lemma (4.3), and the following observation that an anisotropic object is an isotropy torsor in the slice topos over its orbit object.

Lemma 4.15. The unit map $X \stackrel{\varepsilon}{\rightarrow} \psi^{*} \psi_{!}(X)=O_{X}$ of an anisotropic object $X$ of a topos $\mathscr{E}$ is an isotropy torsor of $\mathscr{E} / O_{X}$.

Proof. For any object $X$, the image of $\theta_{X}$ paired with the projection is isomorphic to the pullback $X \times_{O} X$ of $\varepsilon$ with itself, as in the diagram below (left), where $O=O_{X}$.
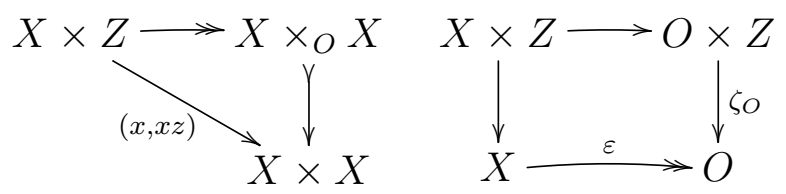

If $X$ is anisotropic, then the epimorphism factor is an isomorphism. The isotropy group $\zeta_{O}$ of $\mathscr{E} / O$ is the projection $O \times Z \rightarrow O$ because $O$ is isotropically trivial. The product $\varepsilon \times \zeta_{O}$ in $\mathscr{E} / O$ is therefore the pullback square in the diagram (right), whence $\varepsilon \times \zeta_{O} \cong \varepsilon \times \varepsilon$ in $\mathscr{E} / O$, so that $\varepsilon$ an isotropy torsor. 
As always, throughout $Z$ denotes the isotropy group of a topos $\mathscr{E}$.

Theorem 4.16. The following conditions on a topos $\mathscr{E}$ are equivalent:

(i) $\mathscr{E}$ is locally anisotropic;

(ii) the isotropy quotient has an étale local section on a globally supported isotropically trivial object $O$.

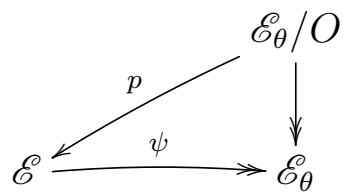

In this case, $O$ is anisotropic (in $\mathscr{E}_{\theta}$ ), and we have

$$
\mathscr{E} / O \simeq \mathcal{B}\left(\mathscr{E}_{\theta} / O ; p^{*} Z\right)
$$

(iii) there is a globally supported anisotropic object $O$ of $\mathscr{E}_{\theta}$, a group bundle

$$
\coprod G_{x} \stackrel{\xi}{\longrightarrow} O \longrightarrow 1
$$

internal to $\mathscr{E}_{\theta}$, and an isotropically trivial étale surjection $\varphi$ such that

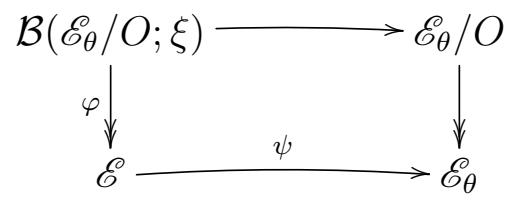

is a topos pullback;

(iv) $\mathscr{E}$ has a globally supported isotropically trivial object $O$ such that

$$
\mathscr{E} / O \simeq \mathcal{B}(\mathscr{F} ; G)
$$

where $G$ is a group internal to an anisotropic topos $\mathscr{F}$.

Proof. These statements all follow from Theorem 4.12, Lemmas 4.15 and 4.3.

Example 4.17. The group classifier Set[Grp], which is the topos of covariant functors $\mathrm{fpGrp} \longrightarrow$ Set on the category fpGrp of finitely presented groups, is not locally anisotropic. In fact, its only anisotropic object is the initial one 0 . Steve Awodey observes, by a general argument about classifying toposes and isotropy groups, that the isotropy group of a topos is its group of model automorphisms of the generic model: the generic model of Set[Grp] is the underlying group functor $U(G)=G$, so that its isotropy group is the group of group automorphisms of $U$. However, one can do even better: George Bergman [1] proves that 
the inner automorphism map from $U$ to its automorphism group is an isomorphism. ${ }^{1}$ Thus, the isotropy group of the group classifier coincides with the generic group $U$. The universal action of $U$ on an object $X: \mathrm{fpGrp} \longrightarrow$ Set is given by

$$
X(G) \times G \rightarrow X(G) ; \quad(x, g) \mapsto X\left(\iota_{g}\right)(x)
$$

where $\iota_{g}$ denotes the inner automorphism of $G$ associated with $g$. We argue that if $X$ is anisotropic, then $X=0$. Indeed, if $A$ is any (finitely presented) Abelian group, then any $A \stackrel{\iota_{g}}{\rightarrow} A$ is the identity, whence the action $X(A) \times A \rightarrow X(A)$ is trivial. Therefore, if $X$ is anisotropic, then $X(A)=\emptyset$ for $A$ non-trivial. But then also $X(1)=\emptyset$ because of the homomorphism $1 \rightarrow 2$, so that $X(G)=\emptyset$ for any group $G$.

\subsection{Isotropy algebras}

We feel it is worthwhile to further explain our results in terms of a concept we shall call an isotropy algebra over a topos. For example, we gain another perspective on Theorem 4.16 in terms of free isotropy algebras. An isotropy algebra over a topos $\mathscr{E}$ is an equivalent formulation of a discrete fibration on the (isotropy) topos groupoid of $\mathscr{E}$ (Remark 4.19).

Definition 4.18. An isotropy algebra $(\phi, s)$ over a topos $\mathscr{E}$ is an atomic geometric morphism $\phi: \mathscr{F} \longrightarrow \mathscr{E}$ together with a section $s$ of the comparison homomorphism $m_{\phi}$ of $\phi$ (8).

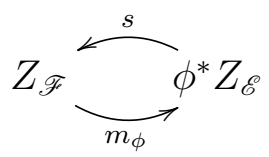

A morphism of isotropy algebras $\tau:(\phi, s) \longrightarrow(\psi, t)$ is an atomic geometric morphism $\tau$ over $\mathscr{E}$
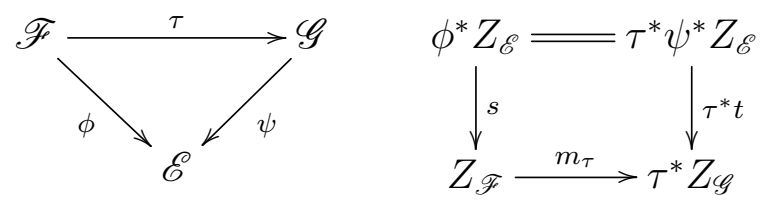

such that the square above (right) in $\mathscr{F}$ commutes.

Remark 4.19. Isotropy algebras over a topos $\mathscr{E}$ are the algebras of a monad on (atomic) geometric morphisms with codomain $\mathscr{E}$. In order to describe this monad we must to revisit the (isotropy) topos groupoid of $\mathscr{E}[5]$ : this is a groupoid internal to the 2-category of (Grothendieck) toposes and geometric morphisms. The object topos is the given topos $\mathscr{E}$, and the morphism topos is $\mathcal{B}(\mathscr{E} ; Z)$. The codomain and identity maps are given by

\footnotetext{
${ }^{1}$ Bergman's article and its consequences for isotropy theory were brought to our attention by Richard Garner in a private correspondence.
} 
the usual structure morphisms $\gamma$ and $u$, and the domain map is given by the universal action $\theta$ as a geometric morphism.

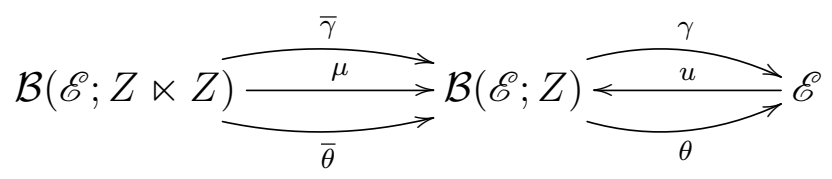

The maps $\bar{\gamma}$ and $\bar{\theta}$ are the projection maps of the following topos pullback.

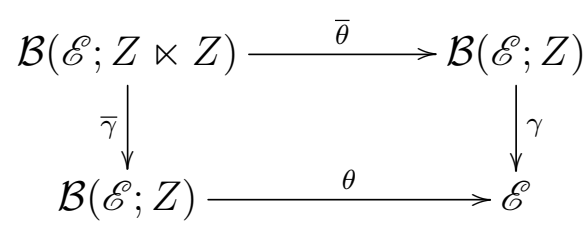

In terms of inverse image functors, $\gamma^{*}(E)$ is $E$ equipped with the trivial action, $u^{*}(E, \sigma)=$ $E$, and $\theta^{*}(E)=\left(E, \theta_{E}\right)$. Something we previously overlooked is that these definitions do indeed define a groupoid: the 'inverse' geometric morphism

$$
\iota: \mathcal{B}(\mathscr{E} ; Z) \longrightarrow \mathcal{B}(\mathscr{E} ; Z)
$$

is given by $\iota^{*}(X, \sigma)=\left(X, \sigma^{\prime}\right)$, such that

$$
\sigma^{\prime}(x, z)=\sigma\left(x z, z^{-1}\right)=\sigma\left(x, z^{-1}\right) z .
$$

It follows from Prop. 6.11 [5], and a thorough discussion of crossed toposes, that the category of isotropy algebras over $\mathscr{E}$ is equivalent to the category of atomic discrete fibrations on the topos groupoid. The monad in question is the 'pullback along codomain and compose with domain' monad associated with the topos groupoid. We omit further details.

Proposition 4.20. The canonical morphism

$$
\theta ! \gamma^{*} E \rightarrow \psi^{*} \psi_{!} E
$$

is an isomorphism for every object $E$ of $\mathscr{E}$. In other words, the so-called Beck-Chevalley condition holds in the following commutative square.

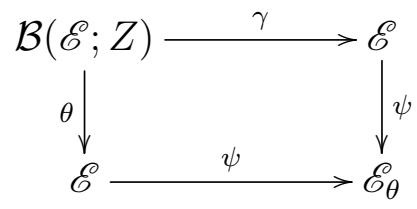


Proof. For any object $(E, \sigma)$ of $\mathcal{B}(\mathscr{E} ; Z), \theta_{!}(E, \sigma)$ is the following coequalizer:

$$
E \times Z \underbrace{\stackrel{\theta_{E}}{\longrightarrow}}_{\sigma_{E}} E \longrightarrow \theta_{!}(E, \sigma) .
$$

If $\sigma$ is the trivial action, then this coequalizer is precisely $\psi_{!}(E)$.

The free isotropy algebra on an object $X$ of a topos $\mathscr{E}$ is the atomic geometric morphism $\widehat{X}$ depicted in the following diagram.

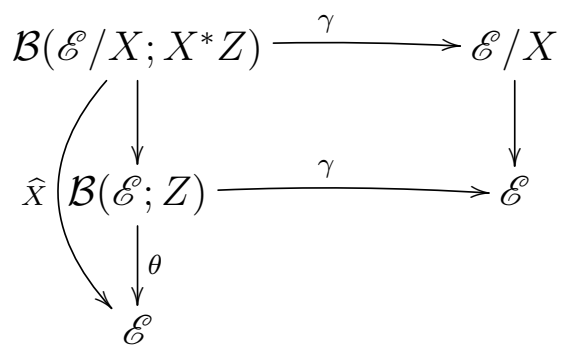

Note that the pullback $\mathcal{B}\left(\mathscr{E} / X ; X^{*} Z\right)$ is equivalent to $\mathcal{B}(\mathscr{E} ; Z) / \gamma^{*} X$. We have $\widehat{X}^{*} Z \cong$ $\overline{X^{*} Z}$, by which we mean the group $X^{*} Z$ with conjugation action (Eg. 3.1). The canonical comparison homomorphism $m$ for $\widehat{X}$, and its section $s$ are given as follows:

$$
\zeta_{X} \ltimes X^{*} \underset{m}{\overleftrightarrow{Z}} \overline{X^{*} Z} \quad ; m(x, z, w)=(x, z w) ; s(x, w)=(x, u, w)
$$

where $1 \stackrel{u}{\rightarrow} Z$ is the unit of $Z$.

Observe that $\widehat{X}_{!}(1)=\theta_{!} \gamma^{*} X$. For any object $X$ of $\mathscr{E}$, let $O_{X}$ denote $\psi^{*} \psi_{!}(X)$ as an object of $\mathscr{E}$. We may apply $\gamma^{*}$ to the unit $X \rightarrow O_{X}$

$$
\gamma^{*} X \rightarrow \gamma^{*} O_{X}=\theta^{*} O_{X},
$$

and then transpose under $\theta_{!} \dashv \theta^{*}$ to give a morphism

$$
\widehat{X}_{!}(1)=\theta_{!} \gamma^{*} X \rightarrow O_{X} .
$$

This morphism is the isomorphism (22).

Theorem 4.21 explains how free isotropy algebras are related to the structure of locally anisotropic toposes. We say that an atomic geometric morphism is isotropically trivial if its canonical comparison homomorphism (8) is an isomorphism.

Theorem 4.21. For any object $X$ of $\mathscr{E}$, the following conditions are equivalent:

(i) $X$ is anisotropic; 
(ii) $\widehat{X}$ is isotropically trivial;

(iii) the following two diagrams are equivalent, where $O_{X}=\widehat{X}_{!}(1)$.
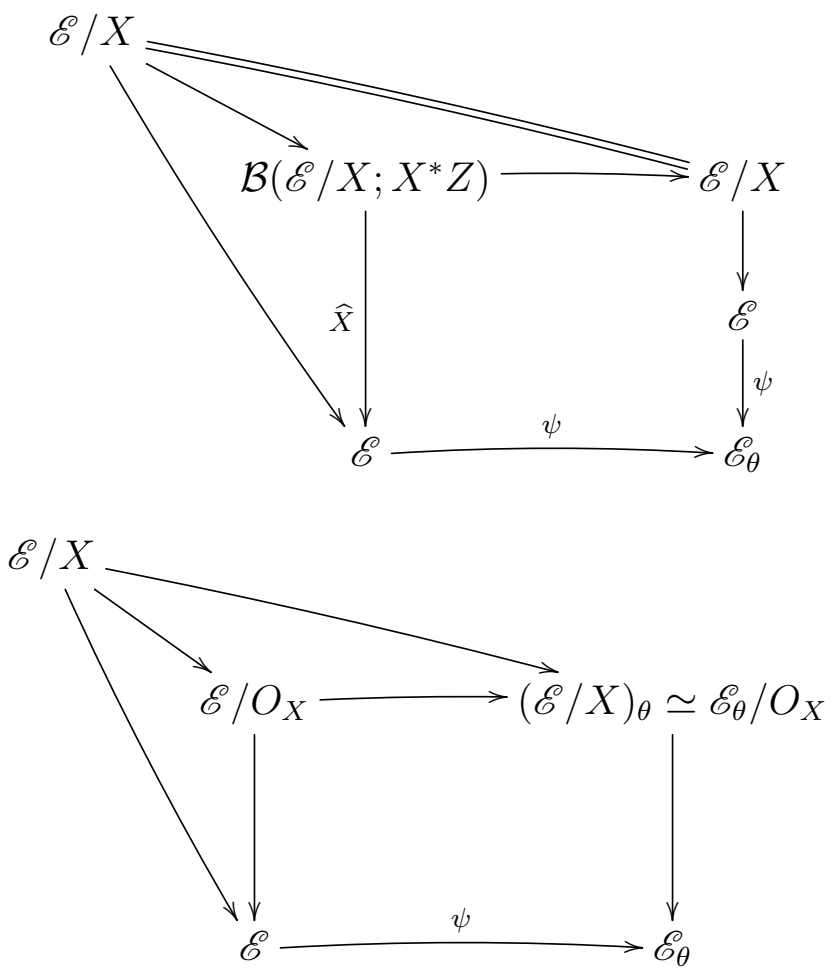

In this case, the square in the first diagram is therefore a pullback, $\widehat{X}$ is étale, and the squares in the two diagrams are equivalent to (21). Moreover, $\mathscr{E}_{\theta} / O_{X}$ is anisotropic.

Proof. If $X$ is anisotropic, then $\zeta_{X}$ is trivial, so that $m$ (24) is an isomorphism. Therefore, (i) implies (ii). Conversely, if $m$ is an isomorphism, then $u$ must be its inverse. Therefore, for all $x \in X$ and $z \in Z$, if $x z=x$, then

$$
(x, z, u)=u m(x, z, u)=u(x, z u)=(x, u, z),
$$

so that $z=u$. Thus, $X$ is anisotropic.

Condition (iii) follows from (i) by Theorem 4.12. Finally, (iii) implies (i) because if the two diagrams are equivalent, then

$$
\mathscr{E} / X \longrightarrow \mathscr{E} \stackrel{\psi}{\longrightarrow} \mathscr{E}_{\theta}
$$

is étale, whence $X$ is anisotropic. 


\section{Inverse semigroups}

The general theory for toposes that we have developed is particularly suited, at least from an algebraic point of view, for a topos coming from an inverse semigroup because such a topos is locally anisotropic.

\subsection{The topos of an inverse semigroup}

Let $S$ be an inverse semigroup with idempotent subset $E=E(S)$. Lawson [9] explains the semigroup notions and terminology we use. We sometimes write an element $s \in S$ as $d \stackrel{s}{\rightarrow} e$, meaning that $s^{*} s=d$ and $s s^{*}=e$, to remind ourselves that we are working in the (Ehresmann) ordered groupoid $G(S)$ associated with $S$. The object poset of $G(S)$ is $E$ and its morphism poset is $S$.

The topos $\mathcal{B}(S)$ of ordered $G(S)$-sets has a somewhat simpler description as follows. A typical object is a right $S$-set $X$, whose action we denote $(x, s) \mapsto x s$, and a function $X \stackrel{p}{\rightarrow} E$ satisfying $x p(x)=x$ and $p(x s)=s^{*} p(x) s$. We refer to $p$ as an étale $S$-set. A morphism is a commutative triangle

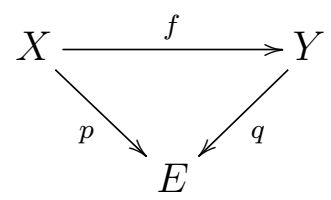

where $f$ is $S$-equivariant. The literature $[4,8,3]$ further explains the topos $\mathcal{B}(S)$, as well as alternative descriptions of it.

Example 5.1. An important étale $S$-set is the 'representable' one associated with an idempotent $e$ :

$$
e: e S \rightarrow E ; t \mapsto t^{*} t
$$

where $e S=\{t \in S \mid e t=t\}$. For any étale $S$-set $X \stackrel{p}{\rightarrow} E$, morphisms $e \rightarrow p$ of $\mathcal{B}(S)$ are in bijection with the fiber $p^{-1}(e)$.

Let $Z(E)=\{s \mid \forall e$, se $=e s\}$ denote the centralizer of the idempotents in $S$. We also have

$$
Z(E)=\left\{s \mid \forall e \leq s s^{*}, s^{*} e s=e\right\}=\left\{s \mid \forall e \leq s^{*} s, s^{*} s^{*}=e\right\} .
$$

The map

$$
\zeta: Z(E) \rightarrow E ; \quad \zeta(s)=s^{*} s=s s^{*},
$$

is the isotropy group of $\mathcal{B}(S)$. The right action by $S$ on $Z(E)$ is given by conjugation: $s \cdot t=t^{*} s t$. The universal action $\theta_{p}$ of $\zeta$ on an étale $S$-set $X \stackrel{p}{\rightarrow} E$ is given by $\theta_{p}(x, s)=x s$, where $x s$ denotes the acton by $S$ on $X$. 
Example 5.2. Another important étale $S$-set is the domain object (or Schützenberger object)

$$
\partial_{0}: S \rightarrow E ; \partial_{0}(s)=s^{*} s .
$$

This object is the generic $S$-torsor, which is a suitable generalization of group torsor, in the sense classified by $\mathcal{B}(S)$ [4]. From this perspective, the isotropy group $\zeta$ of $\mathcal{B}(S)$ may also be regarded as the group of torsor automorphisms of $\partial_{0}$.

A group $G$ internal to the topos $\mathcal{B}(S)$ is equivalently given as a presheaf of groups on $E$ with an action by $S$. Let us refer to $G$ as an étale $S$-group. The associated semidirect product $S \ltimes G$ is itself an inverse semigroup, and the topos $\mathcal{B}(S ; G)$ of right $G$-objects over $\mathcal{B}(S)$ is equivalent to $\mathcal{B}(S \ltimes G)$.

We pass from a prehomomorphism $S \stackrel{\rho}{\rightarrow} T$ between inverse semigroups to a geometric morphism

$$
\rho: \mathcal{B}(S) \longrightarrow \mathcal{B}(T)
$$

whose inverse image functor $\rho^{*}$ carries an étale $S$-set to its pullback along

$$
\rho_{\mid E}: E(S) \rightarrow E(T)
$$

The action by $S$ on the pullback is given by restriction along $\rho$. We refer to $\rho^{*}$ as

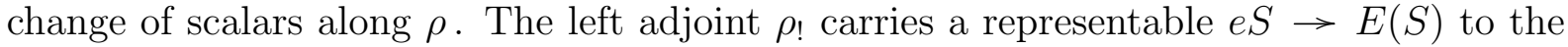
representable $\rho(e) T \rightarrow E(T)$.

The isotropy quotient

$$
\psi: \mathcal{B}(S) \longrightarrow \mathcal{B}(S)_{\theta}
$$

comes from the maximum idempotent-separating congruence, denoted $\varepsilon$ in the following diagram.

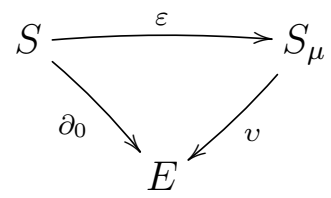

In other words, the inclusion of the isotropically trivial objects in $\mathcal{B}(S)$ is identified with the change of scalars functor $\varepsilon^{*}$. Incidentally, the inverse semigroup $S_{\mu}$ has the same idempotent $\wedge$-semilattice $E$. Moreover, the object $v$ is the orbit space $\psi_{!}\left(\partial_{0}\right)=O \partial_{\partial_{0}}$.

\subsection{Isotropy torsors over an inverse semigroup}

Interpreting Definition 4.8 for $\mathscr{E}=\mathcal{B}(S)$, an isotropy torsor of an inverse semigroup $S$ is an étale $S$-set $X \stackrel{p}{\rightarrow} E$ for which its universal action $\theta_{p}$ is free and transitive. In other words, it is a globally supported $p$ such that the map

$$
p \times \zeta \rightarrow p \times p ;(x, s) \mapsto(x, x s),
$$


is an isomorphism. We also use other terminology: we say that $p$ is anisotropic if this map is injective, which holds if and only if for all $x \in X$ and $s \in Z(E)$ such that $p(x)=s s^{*}$, we have

$$
x s=x \Rightarrow s=p(x) .
$$

We also say that $p$ is connected if $p$ has global support and (26) is surjective.

By Prop. 4.11, isotropy torsors of an inverse semigroup $S$ and étale sections of the isotropy quotient of $\mathcal{B}(S)$ form equivalent groupoids. Thus, isotropy torsors and étale sections of the change of scalars functor $\varepsilon^{*}$ are equivalent. Our goal is to show that this characterization is realized at the semigroup level as certain isomorphism classes of sections of the maximum idempotent-separating quotient $\varepsilon(25)$.

Definition 5.3. A 2-cell

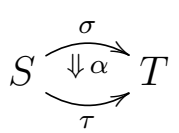

of prehomomorphisms is an order-preserving function $E(S) \stackrel{\alpha}{\rightarrow} T$ such that for all $d \stackrel{s}{\rightarrow} e$ in $S$ the square

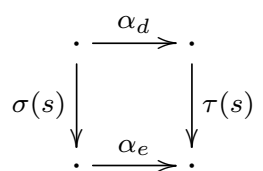

commutes in $G(S): \alpha_{e} \sigma(s)=\tau(s) \alpha_{d}$. Implicitly, also $\alpha_{d}^{*} \alpha_{d}=\sigma(d)$, and so on. Moreover, $\alpha$ is called central if it factors through the centralizer subsemigroup $Z(E) \subseteq T$.

Observe that a 2-cell between prehomomorphisms so defined is invertible such that $\left(\alpha^{-1}\right)_{d}=\alpha_{d}^{*}$. A 2-cell $\alpha$ is central if and only if the whiskering diagram

$$
S \underset{\tau}{\stackrel{\sigma}{\Downarrow \alpha}} T \stackrel{\varepsilon}{\longrightarrow} T_{\mu}
$$

commutes, which means that $\varepsilon \alpha$ equals the identity $\varepsilon \sigma=\varepsilon \tau$. A central 2-cell of prehomomorphisms passes to a natural isomorphism of ordered functors over $G\left(T_{\mu}\right)$
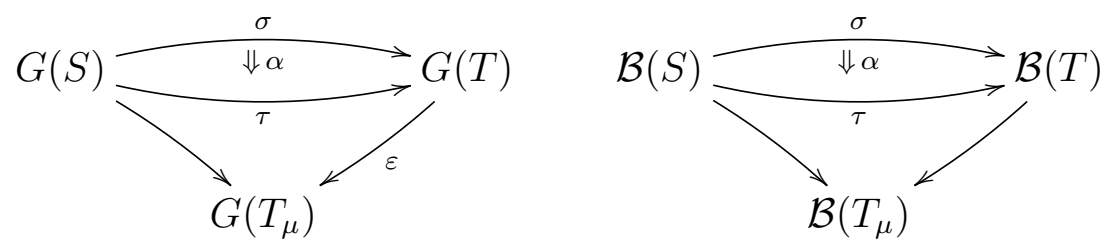

and whence to a natural isomorphism of geometric morphisms over $\mathcal{B}\left(T_{\mu}\right)$ above (right). Equivalently, this happens precisely when the diagram

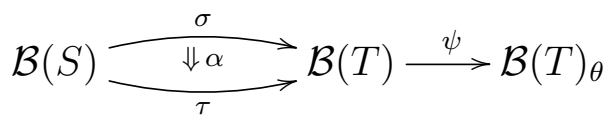


is an equality.

Lemma 5.4. Suppose that $X \stackrel{p}{\rightarrow} E$ is an isotropy torsor of $S$. Then for any element $e S \stackrel{x}{\rightarrow} X($ i.e., $p(x)=e)$ the square

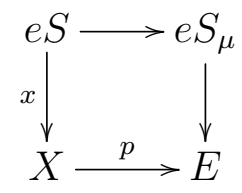

is the adjunction square for $\psi_{!} \dashv \psi^{*}$ associated with $x$. Therefore, it is a pullback (Cor. $4.5)$.

We are ready to state and prove our characterization of isotropy torsors of an inverse semigroup in terms of sections of the maximum idempotent-separating congruence $\varepsilon$.

Theorem 5.5. For an inverse semigroup $S$ the following three groupoids are equivalent:

(i) the groupoid of isotropy torsors of $S$;

(ii) the groupoid of étale sections of the isotropy quotient $\psi$; and

(iii) the groupoid of homomorphic sections of $\varepsilon$ and central isomorphisms between them.

Proof. The equivalence of (i) and (ii) is simply Theorem 4.12. From a homomorphic section of $\varepsilon$, we obtain an étale section of $\psi$ simply in the usual functorial way of assigning a geometric morphism to a prehomomorphism.

Let $X \stackrel{p}{\rightarrow} E$ be an isotropy torsor of $S$. We construct a homomorphic section $\rho$ of $\varepsilon$ by 'coordinatizing' $p$ : we mean by fixing a set-theoretic section $x_{e}$ of $p$. (The section $x_{e}$ cannot be expected to be $S$-equivariant, but that is not necessary.) If $d \stackrel{\bar{s}}{\rightarrow} e$ is an element of $S_{\mu}$, then the pullback diagram

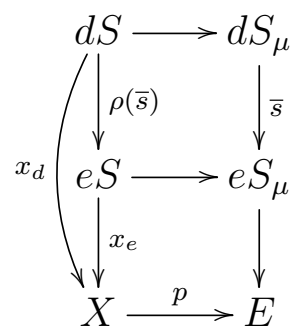

defines $\rho(\bar{s})$. It follows that $\rho$ preserves the restricted product, and because $\varepsilon$ is an idempotent-separating homomorphism, $\rho(d e)=\rho(d) \rho(e)$ holds, so that $\rho$ is indeed a homomorphism. If $\rho_{x}$ is the homomorphism so obtained from a section $E \stackrel{x}{\rightarrow} X$, and $\rho_{y}$ the one obtained from another section $y$, then there is a unique order preserving map $E \stackrel{\alpha}{\rightarrow} Z(E)\left(\alpha(e)^{*} \alpha(e)=e\right)$ such that $x \alpha=y$ and $\alpha_{e} \rho_{y}(\bar{s})=\rho_{x}(\bar{s}) \alpha_{d}$, for all $d \stackrel{\bar{s}}{\rightarrow} e$. This defines a central isomorphism $\alpha: \rho_{y} \Rightarrow \rho_{x}$. The remaining details are straightforward. 
Corollary 5.6. An inverse semigroup $S$ has an isotropy torsor if and only if $S \cong T \ltimes G$, where $G$ is an étale T-group for a fundamental inverse semigroup $T$.

Proof. A semigroup $T \ltimes G$ has an isotropy torsor because $\mathcal{B}(T \ltimes G)$ is equivalent to $\mathcal{B}(\mathscr{F} ; G)$, for $\mathscr{F}=\mathcal{B}(T)$. Conversely, if $S$ has an isotropy torsor, then by Theorem 5.5 its quotient $S \stackrel{\varepsilon}{\rightarrow} S_{\mu}$ has a section $\rho$. By Billhardt's theory [9], we have $S \cong S_{\mu} \ltimes G$, where $G=\rho^{*}(\zeta)$ is an étale $S_{\mu}$-group; $G$ is the isotropy group $\zeta$ regarded as an étale $S_{\mu}$-set by restriction along $\rho$.

\subsection{The idempotent centralizer revisited}

We conclude our explorations by interpreting the structure theorem for locally anisotropic toposes (Theorem 4.16) for inverse semigroups.

Example 5.7. The domain object $S \stackrel{\partial_{0}}{\rightarrow} E$ is anisotropic: if $t^{*} t=s s^{*}$ and $t s=t$, then $s=s s^{*} s=t^{*} t s=t^{*} t=\partial_{0}(t)$. In fact, this shows that $\partial_{0}$ satisfies the stronger "torsion free" property [7]: $\forall x \in X, s \in S$ such that $p(x)=s s^{*}$, we have $x s=x \Rightarrow s=p(x)$.

We have $\mathcal{B}(S) / \partial_{0} \simeq \mathrm{Set}^{E^{\text {op }}}$, and diagram (23) is equivalent to the following one.

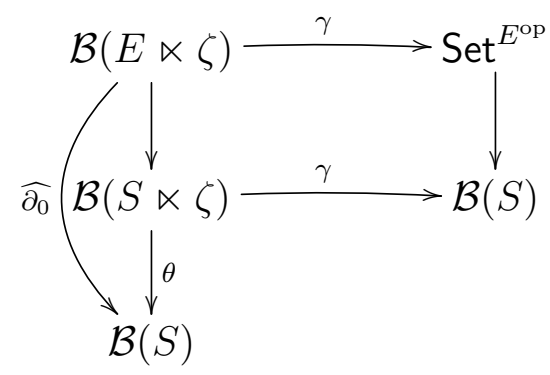

The topos $\mathcal{B}(E \ltimes \zeta)$ is equivalent to $\mathcal{B}(Z(E))$, identifying $\widehat{\partial}_{0}$ with the geometric morphism induced by the inverse subsemigroup

$$
Z(E) \subseteq S .
$$

In other words, the semigroup homomorphism $(27)$ is the free isotropy algebra $\widehat{\partial_{0}}$. Following our notation from $\S 4.2$, we write $O=O_{\partial_{0}}$ for the orbits of $\partial_{0}$ under the action of the isotropy group $\zeta$ (25). We interpret Theorem 4.16 as follows, taking for $X$ the globally supported anisotropic object $\partial_{0}$.

Theorem 5.8. We have topos equivalences

$$
\mathcal{B}(S) / O \simeq \mathcal{B}(E \ltimes \zeta) \simeq \mathcal{B}(Z(E)),
$$

where the isotropy group $\zeta$ is regarded as a presheaf of groups

$$
\zeta(e)=\left\{s \in Z(E) \mid s^{*} s=e\right\}
$$


on E. Furthermore, the two topos pullback squares

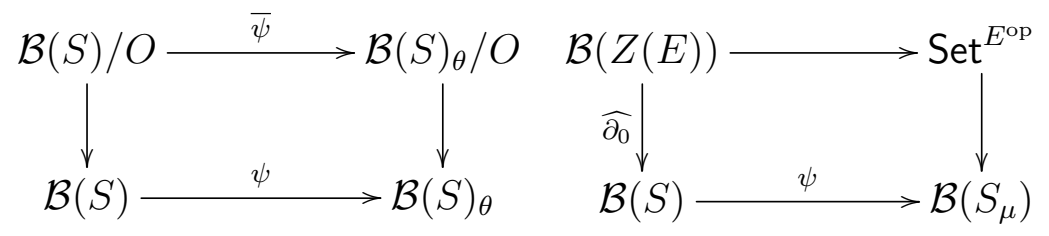

are equivalent, identifying $\widehat{\partial}_{0}$ with $\mathcal{B}(S) / O \longrightarrow \mathcal{B}(S)$. In particular, $\widehat{\partial}_{0}$ is étale and isotropically trivial.

The maximum idempotent-separating quotient $\varepsilon(25)$ is an isotropy torsor regarded as an object of $\mathcal{B}(S) / O$. Furthermore, under the equivalence $\mathcal{B}\left(S_{\mu}\right) / O \simeq \operatorname{Set}^{E^{\text {op }}}$ the group $\bar{\psi}_{*}\left(\varepsilon^{\varepsilon}\right)$ is associated with $\zeta$.

Finally, the equivalence $\mathcal{B}(S) / O \simeq \mathcal{B}(Z(E))$ can be seen explicitly. Indeed, the morphism $E \stackrel{\eta}{\rightarrow} S_{\mu}$ such that $\eta(e)=\varepsilon(e)$ is $Z(E)$-equivariant because for any $s \in Z(E)$ we have

$$
\eta(e \cdot s)=\eta\left(s^{*} e s\right)=\varepsilon\left(s^{*} e s\right)=\varepsilon\left((e s)^{*} e s\right)=\varepsilon(e s)=\varepsilon(e) \cdot s=\eta(e) \cdot s .
$$

An object of $\mathcal{B}(S) / O$ is an $S$-equivariant map $Y \stackrel{f}{\rightarrow} S_{\mu}$ such that $y f(y)^{*} f(y)=y$. We pass such an object $f$ to $\mathcal{B}(Z(E))$ by pullback along $\eta$ : this is indeed an object of $\mathcal{B}(Z(E))$ because $\eta$ is $Z(E)$-equivariant. For instance, the isotropy torsor $\varepsilon$ passes to $Z(E) \rightarrow E$.

\section{Concluding remarks}

We include a list of some problems and questions that we have left for future work.

Functoriality of the isotropy group. The behaviour of isotropy across a geometric morphism is an important aspect that we shall treat elsewhere. In this article we used the canonical comparison map of isotropy groups associated with a locally connected geometric morphism. In general, we find that an arbitrary geometric morphism $\psi: \mathscr{F} \longrightarrow \mathscr{E}$ has associated with it a canonical span of group homomorphisms (perhaps thought of as a bimodule) comparing the isotropy groups of the two toposes.

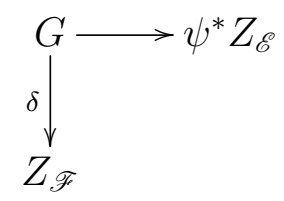

The homomorphism $\delta$ is a crossed module. In the case that $\psi$ is locally connected, $\delta$ is an isomorphism.

Crossed sheaves. The topos of crossed sheaves $\mathscr{E} / Z$ (§ 3.3) is interesting because for one thing it carries a (balanced) braided monoidal closed structure induced by the multiplication on $Z$. Freyd and Yetter [2] study the particular example of crossed $G$-sets for a 
discrete finite group $G$, showing how the resulting monoidal structure is relevant to the study of knot invariants. The topos $\mathscr{E} / Z$ admits a connected atomic quotient to what we call the topos of unital crossed sheaves: those crossed sheaves $X \stackrel{t}{\rightarrow} Z$ for which $x t(x)=x$, for all $x \in X$. In a forthcoming article we prove that this topos inherits a monoidal closed structure, which we conjecture is the universal solution to making the braided structure on $\mathscr{E} / Z$ symmetric.

General structure theorems. We have obtained structural results for toposes whose isotropy quotient has a local étale section. Without the existence of a section it seems difficult to obtain a concrete description of $\mathscr{E}$ as a topos structured over $\mathscr{E}_{\theta}$.

Split isotropy. Remark 4.14 explains that if the isotropy quotient $\mathscr{C} \rightarrow \mathscr{C}_{\theta}$ of a small category $\mathscr{C}$ has a section $p$, then under certain conditions on $\mathscr{C}$ we may obtain a description of $\mathscr{C}$ as a semidirect product $\mathscr{C}_{\theta} \ltimes p^{*} Z$. This suggests an analysis which is analogous to the classification theory for group extensions, aiming for an invariant whose elements correspond to obstructions to the splitting of the quotient.

[1] G. M. Bergman. An inner automorphism is only an inner automorphism, but an inner endomorphism can be something strange. Publicacions Matematiques, 56:91$126,2012$.

[2] P. Freyd and D. Yetter. Braided compact closed categories with applications to low dimensional topology. Advances in Mathematics, 77:156-182, 1989.

[3] J. Funk. Semigroups and toposes. Semigroup Forum, 75(3):480-519, 2007.

[4] J. Funk and P. Hofstra. Topos theoretic aspects of semigroup actions. Theory and Applications of Categories, 24(6):117-147, 2010.

[5] J. Funk, P. Hofstra, and B. Steinberg. Isotropy and crossed toposes. Theory and Applications of Categories, 26(24):660-709, 2012.

[6] P. T. Johnstone. Sketches of an Elephant: A Topos Theory Compendium. Clarendon Press, Oxford, 2002.

[7] A. Kock and I. Moerdijk. Presentations of étendues. Cahiers de Top. et Géom. Diff. Catégoriques, 32(2):145-164, 1991.

[8] M. Lawson and B. Steinberg. Etendues and ordered groupoids. Cahiers de Top. et Géom. Diff. Catégoriques, 40(2):127-140, 2002.

[9] M. Lawson. Inverse Semigroups: The Theory of Partial Symmetries. World Scientific Publishing Co., Singapore, 1998. 
[10] S. Mac Lane and I. Moerdijk. Sheaves in Geometry and Logic. Springer-Verlag, Berlin-Heidelberg-New York, 1992. 